Submitted to the Annals of Statistics

\title{
A GENERAL APPROACH FOR CURE MODELS IN SURVIVAL ANALYSIS
}

\author{
By Valentin Patilea and Ingrid Van Keilegom \\ Univ Rennes, Ensai, CNRS, CREST-UMR 9194, F-35000 Rennes, France \\ ORSTAT, KU Leuven, Belgium
}

\begin{abstract}
In survival analysis it often happens that some subjects under study do not experience the event of interest; they are considered to be 'cured'. The population is thus a mixture of two subpopulations : the one of cured subjects, and the one of 'susceptible' subjects. We propose a novel approach to estimate a mixture cure model when covariates are present and the lifetime is subject to random right censoring. We work with a parametric model for the cure proportion, while the conditional survival function of the uncured subjects is unspecified. The approach is based on an inversion which allows to write the survival function as a function of the distribution of the observable variables. This leads to a very general class of models, which allows a flexible and rich modeling of the conditional survival function. We show the identifiability of the proposed model, as well as the consistency and the asymptotic normality of the model parameters. We also consider in more detail the case where kernel estimators are used for the nonparametric part of the model. The new estimators are compared with the estimators from a Cox mixture cure model via simulations. Finally, we apply the new model on a medical data set.
\end{abstract}

1. Introduction. Driven by emerging applications, over the last two decades there has been an increasing interest for time-to-event analysis models allowing the situation where a fraction of the right censored observed lifetimes corresponds to subjects who will never experience the event. In biostatistics such models including covariates are usually called cure models and they allow for a positive cure fraction, or cure rate, that corresponds to the proportion of patients cured of their disease. For a review of these models in survival analysis, see for instance Peng \& Taylor (2014) or Amico \& Van Keilegom (2018). Economists sometimes call such models split pop-

MSC 2010 subject classifications: Primary 62N01, 62N02; secondary 62F12, 62 G05

Keywords and phrases: Asymptotic normality, bootstrap, kernel smoothing, logistic regression, mixture cure model, semiparametric model 
ulation models (see Schmidt \& Witte 1989), while the reliability engineers refer to them as limited-failure population life models (Meeker 1987).

At first sight, a cure regression model is nothing but a binary outcome, cured versus uncured, regression problem. The difficulty comes from the fact that the cured subjects are unlabeled observations among the censored data. Then one has to use all the observations, censored and uncensored, to complete the missing information and thus to identify, estimate and make inference on the cure fraction regression function. We propose a general approach for this task, a tool that provides a general ground for cure regression models. The idea is to start from the laws of the observed variables and to express the quantities of interest, such as the cure rate and the conditional survival of the uncured subjects, as functionals of these laws. These general expressions, that we call inversion formulae and that we derive with no particular constraint on the space of the covariates, are the vehicles that allow for a wide modeling choice, parametric, semiparametric and nonparametric, for both the law of the lifetime of interest and the cure rate. Indeed, the inversion formulae allow to express the likelihood of the binary outcome model as a function of the laws of the observed variables. The maximum likelihood estimator of the parameter vector of the cure fraction function is then simply the maximizer of the likelihood obtained by replacing the laws of the observations by some estimators. With at hand the estimate of the parameter of the cure fraction, the inversion formulae will provide an estimate for the conditional survival of the uncured subjects. For the sake of clarity, we focus on the so-called mixture cure models with a parametric cure fraction function, the type of model that is most popular among practitioners. Meanwhile, the law of the lifetime of interest is left unspecified.

The paper is organized as follows. In Section 2 we provide a general description of the model. Next, we introduce the needed notation and present the inversion formulae on which our approach is built. We finish Section 2 by a discussion of the identification issue and some new insight in the existing approaches in the literature. Section 3 introduces the general maximum likelihood estimator, while in Section 4 we derive the asymptotic results. A simple bootstrap procedure for making feasible inference is proposed. Section 4 ends with an illustration of our approach in the case where the conditional law of the observations is estimated by kernel smoothing. In Section 5 we calculate the efficient score, which is needed for obtaining an efficient estimator of the parameters in the model. In Sections 6 and 7 we report some empirical results obtained with simulated and one real data sets. Our estimator performs well in simulations and provides similar or more interpretable results in applications compared with a competing logistic/proportional hazards 
mixture approach. The proofs of the main results are relegated to the Appendix. Some technical details, additional simulation results and a second real data illustration are collected in an online Supplement.

\section{The model.}

2.1. A general class of mixture cure models. Let $T$ denote (a possible monotone transformation of) the lifetime of interest that takes values in $(-\infty, \infty]$. A cured observation corresponds to the event $\{T=\infty\}$, and in the following this event is allowed to have a positive probability. Let $X$ be a covariate vector belonging to $\mathcal{X}$ a general covariate space. The covariate vector could include discrete and continuous components. The survival function $F_{T}((t, \infty] \mid x)=\mathbb{P}(T>t \mid X=x)$, can be written as

$$
F_{T}((t, \infty] \mid x)=1-\phi(x)+\phi(x) F_{T, 0}((t, \infty) \mid x), \quad t \in \mathbb{R}, x \in \mathcal{X},
$$

where $1-\phi(x)=\mathbb{P}(T=\infty \mid X=x)$ is the cure rate and $F_{T, 0}((t, \infty) \mid x)=$ $\mathbb{P}(T>t \mid X=x, T<\infty)$. Depending on which model is used for $\phi(x)$ and $F_{T, 0}(\cdot \mid x)$, one obtains a parametric, semi- or nonparametric model, called a 'mixture cure model'. In the literature, $\phi(x)$ often follows a logistic model, i.e. $\phi(x)=\exp \left(a+x^{\top} b\right) /\left[1+\exp \left(a+x^{\top} b\right)\right]$ for some $\left(a, b^{\top}\right)^{\top} \in \mathbb{R}^{1+d}$. (Herein, a vector is a column matrix and for any matrix $A, A^{\top}$ denotes its transpose.) Recently, semiparametric models (like a single-index model as in Amico et al. 2018) or nonparametric models (as in Xu \& Peng 2014 or López-Cheda et al. 2017) have been proposed. As for the survival function $F_{T, 0}(\cdot \mid x)$ of the susceptible subjects, a variety of models have been proposed, including parametric models (see e.g. Boag 1949, Farewell 1982), semiparametric models based on a proportional hazards assumption (e.g. Kuk \& Chen 1992, Sy \& Taylor 2000, Fang et al. 2005, Lu 2008; see also Othus et al. 2009) or nonparametric models (e.g. Taylor 1995, Xu \& Peng 2014).

In this paper we propose to model $\phi(x)$ parametrically, i.e. we assume that $\phi(\cdot)$ belongs to the family of conditional probability functions $\{\phi(\cdot, \beta): \beta \in$ $B$, where $\beta$ is the parameter vector of the model and $B$ is the parameter set. This family could be the logistic family or any other parametric family. For the survival function $F_{T, 0}(\cdot \mid x)$ we do not impose any assumptions in order to have a flexible and rich class of models for $F_{T}(\cdot \mid x)$ to choose from.

Later on we will see that for the estimation of $F_{T, 0}(\cdot \mid x)$ any estimator that satisfies certain minimal conditions can be used, and hence we allow for a large variety of parametric, semiparametric and nonparametric methods.

As is often the case with time-to-event data, we assume that the lifetime $T$ is subject to random right censoring, i.e. instead of observing $T$, we only 
observe the pair $(Y, \delta)$, where $Y=T \wedge C, \delta=\mathbf{1}\{T \leq C\}$ and $C$ is a real-valued random variable, called the censoring time. Some identification assumptions are required to be able to identify the conditional law of $T$ from the observed variables $Y$ and $\delta$. Let us assume that

$$
C \perp T \mid X \quad \text { and } \quad \mathbb{P}(C<\infty)=1 .
$$

The conditional independence between $T$ and $C$ is an usual identification assumption in survival analysis in the presence of covariates. The zero probability at infinity condition for $C$ implies that $\mathbb{P}(C<\infty \mid X)=1$ almost surely (a.s.). This latter mild condition is required if we admit that the observations $Y$ are finite, which is the case in the common applications. For the sake of simplicity, let us also consider the commonly used condition

$$
\mathbb{P}(T=C)=0,
$$

which implies that $\mathbb{P}(T=C \mid X)=0$ a.s.

2.2. Some notations and preliminaries. We start with some arguments which are valid without assuming any model on the functions $\phi, F_{T}$ and $F_{C}$. The observations are characterized by the conditional sub-probabilities

$$
\begin{aligned}
& H_{1}((-\infty, t] \mid x)=\mathbb{P}(Y \leq t, \delta=1 \mid X=x) \\
& H_{0}((-\infty, t] \mid x)=\mathbb{P}(Y \leq t, \delta=0 \mid X=x), \quad t \in \mathbb{R}, x \in \mathcal{X} .
\end{aligned}
$$

Then $H((-\infty, t] \mid x) \stackrel{\text { def }}{=} \mathbb{P}(Y \leq t \mid X=x)=H_{0}((-\infty, t] \mid x)+H_{1}((-\infty, t] \mid$ $x)$. Since we assume that $Y$ is finite, we have

$$
H((-\infty, \infty) \mid x)=1, \quad \forall x \in \mathcal{X} .
$$

For $j \in\{0,1\}$ and $x \in \mathcal{X}$, let $\tau_{H_{j}}(x)=\inf \left\{t: H_{j}([t, \infty) \mid x)=0\right\}$ denote the right endpoint of the support of the conditional sub-probability $H_{j}$. Let us define $\tau_{H}(x)$ in a similar way and note that $\tau_{H}(x)=\max \left\{\tau_{H_{0}}(x), \tau_{H_{1}}(x)\right\}$. Note that $\tau_{H_{0}}(x), \tau_{H_{1}}(x)$ and $\tau_{H}(x)$ can equal infinity, even though $Y$ is finite. For $x \in \mathcal{X}$ and $-\infty<t \leq \infty$, we define the conditional probabilities $F_{C}((-\infty, t] \mid x)=\mathbb{P}(C \leq t \mid X=x)$ and $F_{T}((-\infty, t] \mid x)=\mathbb{P}(T \leq t \mid X=x)$.

Let us show how the probability of being cured could be identified from the observations without any reference to a model for this probability. Under conditions (2.2)-(2.3) we can write

$$
H_{1}(d t \mid x)=F_{C}([t, \infty) \mid x) F_{T}(d t \mid x), \quad H_{0}(d t \mid x)=F_{T}([t, \infty] \mid x) F_{C}(d t \mid x),
$$

and $H([t, \infty) \mid x)=F_{T}([t, \infty] \mid x) F_{C}([t, \infty) \mid x)$. These equations could be solved and thus they allow to express the functions $F_{T}(\cdot \mid x)$ and $F_{C}(\cdot \mid x)$ 
in an unique way as explicit transformations of $H_{0}(\cdot \mid x)$ and $H_{1}(\cdot \mid x)$. For this purpose, let us consider the conditional cumulative hazard measures

$$
\Lambda_{T}(d t \mid x)=\frac{F_{T}(d t \mid x)}{F_{T}([t, \infty] \mid x)} \quad \text { and } \quad \Lambda_{C}(d t \mid x)=\frac{F_{C}(d t \mid x)}{F_{C}([t, \infty) \mid x)}, \quad x \in \mathcal{X} .
$$

The model equations yield

$$
\Lambda_{T}(d t \mid x)=\frac{H_{1}(d t \mid x)}{H([t, \infty) \mid x)} \quad \text { and } \quad \Lambda_{C}(d t \mid x)=\frac{H_{0}(d t \mid x)}{H([t, \infty) \mid x)} .
$$

Then, we can write the following functionals of $H_{0}(\cdot \mid x)$ and $H_{1}(\cdot \mid x)$ :

$$
\begin{aligned}
& F_{T}((t, \infty] \mid x)=\prod_{s \leq t}\left\{1-\Lambda_{T}(d s \mid x)\right\}, \\
& F_{C}((t, \infty) \mid x)=\prod_{s \leq t}\left\{1-\Lambda_{C}(d s \mid x)\right\}, \quad t \in \mathbb{R},
\end{aligned}
$$

where $\prod_{s \in A}$ stands for the product-integral over the set $A$ (see Gill and Johansen 1990). Let us point out that

$$
\mathbb{P}(T=\infty \mid x)=\prod_{t \in \mathbb{R}}\left\{1-\Lambda_{T}(d t \mid x)\right\}=\prod_{t \in \mathbb{R}}\left\{1-\frac{H_{1}(d t \mid x)}{H([t, \infty) \mid x)}\right\} .
$$

Moreover, if $\tau_{H_{1}}(x)<\infty$, then

$$
\mathbb{P}\left(T>\tau_{H_{1}}(x) \mid x\right)=\prod_{t \in\left(-\infty, \tau_{H_{1}}(x)\right]}\left\{1-\Lambda_{T}(d t \mid x)\right\}
$$

but there is no way to identify the conditional law of $T$ beyond $\tau_{H_{1}}(x)$. Therefore, we will impose

$$
\mathbb{P}\left(T>\tau_{H_{1}}(x) \mid x\right)=\mathbb{P}(T=\infty \mid x),
$$

i.e. $\prod_{t \in \mathbb{R}}\left\{1-\Lambda_{T}(d t \mid x)\right\}=\prod_{-\infty<t \leq \tau_{H_{1}}(x)}\left\{1-\Lambda_{T}(d t \mid x)\right\}$. Note that if $\tau_{H_{1}}(x)=\infty$, condition (2.8) is no longer an identification restriction, but just a simple consequence of the definition of $\Lambda_{T}(\cdot \mid x)$. Finally, the condition that $\mathbb{P}(C<\infty)=1$ in $(2.2)$ can be re-expressed by saying that we assume that $H_{0}(\cdot \mid x)$ and $H_{1}(\cdot \mid x)$ are such that, $\forall x \in \mathcal{X}$,

$$
\mathbb{P}(C=\infty \mid x)=\prod_{t \in \mathbb{R}}\left\{1-\Lambda_{C}(d t \mid x)\right\}=\prod_{t \in \mathbb{R}}\left\{1-\frac{H_{0}(d t \mid x)}{H([t, \infty) \mid x)}\right\}=0
$$


This condition is satisfied only if $\tau_{H_{1}}(x) \leq \tau_{H_{0}}(x)$. Indeed, if $\tau_{H_{1}}(x)>\tau_{H_{0}}(x)$ then necessarily $\tau_{H_{0}}(x)<\tau_{H}(x)$ and so $H\left(\left[\tau_{H_{0}}(x), \infty\right) \mid x\right)>0$. Hence, $\Lambda_{C}(\mathbb{R} \mid x)=\Lambda_{C}\left(\left(-\infty, \tau_{H_{0}}(x)\right] \mid x\right)<\infty$, and thus $\mathbb{P}(C=\infty \mid x)>0$, which contradicts (2.9). It is important to understand that any two conditional sub-probabilities $H_{0}(\cdot \mid x)$ and $H_{1}(\cdot \mid x)$ such that $H_{0}(\mathbb{R} \mid x)+H_{1}(\mathbb{R} \mid x)=1$, $\forall x \in \mathcal{X}$, define uniquely $F_{T}(\cdot \mid x)$ and $F_{C}(\cdot \mid x)$. If $H_{0}(\cdot \mid x)$ and $H_{1}(\cdot \mid x)$ are such that conditions (2.2) and (2.3) hold, then $F_{T}(\cdot \mid x)$ is the probability distribution of $T$ given $X=x$, with all the mass beyond $\tau_{H_{1}}(x)$ concentrated at infinity provided condition $(2.8)$ holds true. In general, $F_{T}(\cdot \mid x)$ and $F_{C}(\cdot \mid x)$ are only functionals of $H_{0}(\cdot \mid x)$ and $H_{1}(\cdot \mid x)$. Finally, note that the vector $X$ gathers all the covariates, but the cure rate and the conditional (sub)distributions $F_{T, 0}, F_{C}, H_{0}$ and $H_{1}$ could depend on different components, on the same or they could only share some common components of $X$. Our methodology allows for any of these situations.

We will assume conditions (2.2), (2.3) and (2.8) throughout the paper.

2.3. A key point for the new approach: the inversion formulae. Write

$$
\begin{aligned}
H([t, \infty) \mid x) & =F_{T}([t, \infty] \mid x) F_{C}([t, \infty) \mid x) \\
& =F_{T}([t, \infty) \mid x) F_{C}([t, \infty) \mid x)+\mathbb{P}(T=\infty \mid x) F_{C}([t, \infty) \mid x),
\end{aligned}
$$

and thus

$$
F_{T}([t, \infty) \mid x)=\frac{H([t, \infty) \mid x)-\mathbb{P}(T=\infty \mid x) F_{C}([t, \infty) \mid x)}{F_{C}([t, \infty) \mid x)}
$$

Consider the conditional cumulative hazard measure for the finite values of the lifetime of interest:

$$
\Lambda_{T, 0}(d t \mid x) \stackrel{\text { def }}{=} \frac{F_{T, 0}(d t \mid x)}{F_{T, 0}([t, \infty) \mid x)}=\frac{F_{T}(d t \mid x)}{F_{T}([t, \infty) \mid x)}
$$

for $t \in \mathbb{R}$. Since $H_{1}(d t \mid x)=F_{C}([t, \infty) \mid x) F_{T}(d t \mid x)$, using (2.10) we obtain

$$
\Lambda_{T, 0}(d t \mid x)=\frac{H_{1}(d t \mid x)}{H([t, \infty) \mid x)-\mathbb{P}(T=\infty \mid x) F_{C}([t, \infty) \mid x)}
$$

Next, using the product-integral we can write

$$
F_{T, 0}((t, \infty) \mid x)=\prod_{s \leq t}\left\{1-\Lambda_{T, 0}(d s \mid x)\right\}, \quad t \in \mathbb{R}, x \in \mathcal{X} .
$$

Note that, by construction, $F_{T, 0}(\mathbb{R} \mid x)=1$.

Let us recall that $F_{C}(\cdot \mid x)$ can be written as a transformation of $H_{0}(\cdot \mid x)$ and $H_{1}(\cdot \mid x)$, see equations (2.5) and (2.6). This representation is not 
surprising since we can consider $C$ as a lifetime of interest and hence $T$ plays the role of a censoring variable. Hence, estimating the conditional distribution function $F_{C}(\cdot \mid x)$ should not be more complicated than in a classical conditional Kaplan-Meier setup, since the fact that $T$ could be equal to infinity with positive probability is irrelevant when estimating $F_{C}(\cdot \mid x)$.

The representations of $F_{C}(\cdot \mid x)$ and $\mathbb{P}(T=\infty \mid x)$ given in equations (2.6) and (2.7), respectively, plugged into equation (2.11), allows to express in a unique way $\Lambda_{T, 0}(\cdot \mid x)$, and thus $F_{T, 0}(\cdot \mid x)$, as maps of the measures $H_{0}(\cdot \mid x)$ and $H_{1}(\cdot \mid x)$. This will be the key element for providing more insight in existing approaches and the starting point of our new approach.

2.4. Model identification issues. Recall that our model involves the functions $F_{T, 0}(\cdot \mid x), F_{C}(\cdot \mid x)$ and $\phi(\cdot, \beta)$, and the assumptions $(2.2),(2.3),(2.8)$.

Let $F_{Y, \delta}(\cdot, \cdot \mid x)$ denote the conditional law of $(Y, \delta)$ given $X=x$. Moreover, let $F_{Y, \delta}^{\beta}(d t, 1 \mid x)=\phi(x, \beta) F_{C}((t, \infty) \mid x) F_{T, 0}(d t \mid x)$, and

$$
F_{Y, \delta}^{\beta}(d t, 0 \mid x)=\left[F_{T, 0}([t, \infty) \mid x) \phi(x, \beta)+1-\phi(x, \beta)\right] F_{C}(d t \mid x) .
$$

These equations define a conditional law for the observations $(Y, \delta)$ based on the model. More precisely, for a choice of $F_{T}(\cdot \mid x), F_{C}(\cdot \mid x)$ and $\beta$, the model yields a conditional law for $(Y, \delta)$ given $X=x$. If the mixture cure model is correctly specified, there exists a value $\beta_{0}$ such that

$$
F_{Y, \delta}(\cdot, \cdot \mid x)=F_{Y, \delta}^{\beta_{0}}(\cdot, \cdot \mid x), \quad \forall x \in \mathcal{X} .
$$

The next question is whether $F_{T, 0}(\cdot \mid x), F_{C}(\cdot \mid x)$ and $\beta_{0}$ are identifiable.

Proposition 2.1. Consider a cure mixture model as in equation (2.1). Assume that conditions (2.2), (2.3), (2.8) are met. Moreover, assume that the cure rate model $\{\phi(\cdot, \beta): \beta \in B\}$ is correct and satisfies the condition

$$
\forall \beta, \widetilde{\beta} \in B \text { such that } \mathbb{P}(\phi(X, \beta)=\phi(X, \widetilde{\beta}))=1 \text {, we have } \beta=\widetilde{\beta} \text {. }
$$

Then $F_{T, 0}(\cdot \mid x), F_{C}(\cdot \mid x)$ and $\beta_{0}$ are identifiable.

2.5. Interpreting the previous modeling approaches. We suppose here that the function $\phi(x)$ follows a logistic model, and comment on several models for $F_{T, 0}$ that have been considered in the literature.

2.5.1. Parametric and proportional hazards mixture model. In a parametric modeling, usually $\tau_{H_{0}}(x)=\tau_{H_{1}}(x)=\infty$ and $\Lambda_{T, 0}(\cdot \mid x)$ belongs to a parametric family of cumulative hazard functions, like for instance the 
Weibull model; see Farewell (1982). Several contributions proposed a more flexible semiparametric proportional hazards $(\mathrm{PH})$ approach; see Fang et al. (2005), Lu (2008) and the references therein. In such a model one imposes a $\mathrm{PH}$ structure for the $\Lambda_{T, 0}(\cdot \mid x)$ measure. More precisely, it is supposed that

$$
\Lambda_{T, 0}(d t \mid x)=\frac{H_{1}(d t \mid x)}{H([t, \infty) \mid x)-\mathbb{P}(T=\infty \mid x) F_{C}([t, \infty) \mid x)}=\exp \left(x^{\top} \gamma\right) \Lambda_{0}(d t),
$$

where $\gamma$ is some parameter to be estimated and $\Lambda_{0}(\cdot)$ is an unknown baseline cumulative hazard function. Our inversion formulae reveal that the parameters $\gamma$ and $\Lambda_{0}$ depend on the conditional measures $H_{0}(\cdot \mid x), H_{1}(\cdot \mid x)$, but these parameters are also connected to the parameter $\beta$ used to model the cure rate $\mathbb{P}(T=\infty \mid x)$. The same is true for the parametric models.

2.5.2. Kaplan-Meier mixture cure model. Taylor (1995) suggested to estimate $F_{T, 0}$ using a Kaplan-Meier type estimator. With such an approach one implicitly assumes that the law of $T$ given $X$ and given that $T<\infty$ does not depend on $X$. This is equivalent to supposing $\Lambda_{T, 0}(\cdot \mid x)=\Lambda_{T, 0}(\cdot)$. Next, to estimate $\Lambda_{T, 0}(\cdot)$ one has to modify the unconditional version of the inversion formulae (2.5) to take into account the conditional probability of the event $\{T=\infty\}$. Following Taylor's approach we rewrite (2.11) as

$$
\Lambda_{T, 0}(d t)=\frac{H_{1}(d t \mid x)}{H_{1}([t, \infty) \mid x)+\int_{[t, \infty)}\left\{1-\frac{1-\phi(x, \beta)}{\phi(x, \beta) F_{T, 0}([s, \infty))+1-\phi(x, \beta)}\right\} H_{0}(d s \mid x)} .
$$

Next, assume that the last equality remains true if $H_{0}(d t \mid x)$ and $H_{1}(d t \mid x)$ are replaced by their unconditional versions, that is assume that

$$
\Lambda_{T, 0}(d t)=\frac{H_{1}(d t)}{H_{1}([t, \infty))+\int_{[t, \infty)}\left\{1-\frac{1-\phi(x, \beta)}{\phi(x, \beta) F_{T, 0}([s, \infty))+1-\phi(x, \beta)}\right\} H_{0}(d s)} .
$$

See equations (2) and (3) in Taylor (1995). The equation above could be solved iteratively by a EM-type procedure: for a given $\beta$ and an iteration $F_{T, 0}^{(m)}(\cdot)$, build $\Lambda_{T, 0}^{(m+1)}(d t)$ and the updated estimate $F_{T, 0}^{(m+1)}(\cdot)$. Let us point out that even if $(T, C)$ is independent of $X$ given that $T<\infty$, the subdistributions $H_{0}(\cdot \mid x)$ and $H_{1}(\cdot \mid x)$ still depend on $x$, since

$$
H_{0}(d t \mid x)=\left\{\mathbb{P}(T<\infty \mid x) F_{T, 0}([t, \infty))+\mathbb{P}(T=\infty \mid x)\right\} F_{C}(d t),
$$

and $H_{1}(d t \mid x)=\mathbb{P}(T<\infty \mid x) F_{C}([t, \infty)) F_{T, 0}(d t)$. Hence,

$$
\Lambda_{T, 0}(d t)=\frac{H_{1}(d t \mid x)}{H_{1}([t, \infty) \mid x)+\int_{[t, \infty)}\left\{1-\frac{1-\phi(x, \beta)}{\phi(x, \beta) F_{T, 0}([s, \infty))+1-\phi(x, \beta)}\right\} H_{0}(d s \mid x)}
$$

would be a more natural form of equation (2.15). The study of an iterative procedure based on this alternative identity will be considered elsewhere. 
3. Maximum likelihood estimation. Let $\left(Y_{i}, \delta_{i}, X_{i}\right)(i=1, \ldots, n)$ be a sample of $n$ i.i.d. copies of the vector $(Y, \delta, X)$. We use a likelihood approach based on formulae (2.6), (2.11) and (2.12) to build an estimator of $\phi(\cdot, \beta)$. To build the likelihood we use estimates $\widehat{H}_{k}(\cdot \mid x)$ of the subdistributions $H_{k}(\cdot \mid x), k \in\{0,1\}$. We consider that for each $x, \widehat{H}(\cdot \mid x)=\widehat{H}_{0}(\cdot \mid$ $x)+\widehat{H}_{1}(\cdot \mid x)$ is a proper distribution. These estimates are constructed with the sample of $(Y, \delta, X)$, without reference to any model for the variables $T$, $C$ or for the conditional probability $\mathbb{P}(T<\infty \mid x)$. At this stage it is not necessary to impose a particular form for $\widehat{H}_{k}(\cdot \mid x)$. One could, for instance, use nonparametric estimators as proposed by Stone (1977) separately for $\delta=1$ and $\delta=0$. To derive the asymptotic results we will only impose that these estimators satisfy some mild conditions.

Let $\widehat{F}_{T, 0}(\cdot \mid x)$ be defined as in equations (2.11) and (2.12) with $\widehat{H}_{0}(\cdot \mid x)$ and $\widehat{H}_{1}(\cdot \mid x)$ instead of $H_{0}(\cdot \mid x)$ and $H_{1}(\cdot \mid x)$, that is $\forall t \in \mathbb{R}, \forall x \in \mathcal{X}$,

$$
\widehat{F}_{T, 0}((t, \infty) \mid x)=\prod_{s \leq t}\left\{1-\frac{\widehat{H}_{1}(d s \mid x)}{\widehat{H}([s, \infty) \mid x)-\mathbb{P}(\widehat{T=\infty} \mid x) \widehat{F}_{C}([s, \infty) \mid x)}\right\}
$$

where

$$
\widehat{F}_{C}((t, \infty) \mid x)=\prod_{s \leq t}\left\{1-\frac{\widehat{H}_{0}(d s \mid x)}{\widehat{H}([s, \infty) \mid x)}\right\}
$$

and the estimator of the cure rate is derived from equation (2.7), i.e.

$$
\mathbb{P}(\widehat{T=\infty} \mid x)=\prod_{t \in \mathbb{R}}\left\{1-\frac{\widehat{H}_{1}(d t \mid x)}{\widehat{H}([t, \infty) \mid x)}\right\} .
$$

By construction, $\forall x, \widehat{F}_{T, 0}(\cdot \mid x)=0$ is a proper distribution function and $\widehat{F}_{T, 0}((t, \infty) \mid x)=0$ for any $t$ such that $\widehat{H}_{1}((t, \infty) \mid x)=0$, provided $\widehat{H}_{1}(\cdot \mid x)$ puts a positive mass at the right endpoint of its support. See Lemma 8.1.

Let $f_{X}$ denote the density of the covariate vector with respect to some dominating measure. The contribution of the observation $\left(Y_{i}, \delta_{i}, X_{i}\right)$ to the likelihood is then $\phi\left(X_{i}, \beta\right) \widehat{F}_{T, 0}\left(\left\{Y_{i}\right\} \mid X_{i}\right) f_{X}\left(X_{i}\right) \widehat{F}_{C}\left(\left(Y_{i}, \infty\right) \mid X_{i}\right)$ when $\delta_{i}=$ 1 , while the contribution when $\delta_{i}=0$ is

$$
\widehat{F}_{C}\left(\left\{Y_{i}\right\} \mid X_{i}\right) f_{X}\left(X_{i}\right)\left[\phi\left(X_{i}, \beta\right) \widehat{F}_{T, 0}\left(\left[Y_{i}, \infty\right) \mid X_{i}\right)+1-\phi\left(X_{i}, \beta\right)\right] .
$$

Since the law of the covariate vector does not carry information on the parameter $\beta$, and since $\widehat{F}_{C}(\cdot \mid x)$ and $\widehat{F}_{T, 0}(\cdot \mid x)$ could be directly computed from $\widehat{H}_{0}(\cdot \mid x)$ and $\widehat{H}_{1}(\cdot \mid x)$, we can drop the factors $\widehat{F}_{T, 0}\left(\left\{Y_{i}\right\} \mid\right.$ 
$\left.X_{i}\right) f_{X}\left(X_{i}\right) \widehat{F}_{C}\left(\left(Y_{i}, \infty\right) \mid X_{i}\right)$ and $\widehat{F}_{C}\left(\left\{Y_{i}\right\} \mid X_{i}\right) f_{X}\left(X_{i}\right)$. Hence the criterion to be maximized with respect to $\beta \in B$ is $\widehat{L}_{n}(\beta)$ where

$$
\widehat{L}_{n}(\beta)=\prod_{i=1}^{n} \phi\left(X_{i}, \beta\right)^{\delta_{i}}\left\{\phi\left(X_{i}, \beta\right) \widehat{F}_{T, 0}\left(\left[Y_{i}, \infty\right) \mid X_{i}\right)+1-\phi\left(X_{i}, \beta\right)\right\}^{1-\delta_{i}} .
$$

The estimator we propose is

$$
\widehat{\beta}=\arg \max _{\beta \in B} \log \widehat{L}_{n}(\beta) .
$$

The existence and the unicity of $\widehat{\beta}$ could be guaranteed using the same conditions one would impose when considering the binary outcome regression model $\{\phi(\cdot, \beta): \beta \in B\}$ and when observing the outcomes.

4. General asymptotic results. Little assumptions were needed so far. To proceed further with the asymptotic results we need to be more specific with respect to several aspects. In order to prove consistency, we have to control the asymptotic behavior of $\widehat{L}_{n}(\beta)$ along sequences of values of the parameter $\beta$. Such a control requires a control of denominators like $\widehat{H}([t, \infty) \mid x)-\mathbb{P}(\widehat{T=\infty} \mid x) \widehat{F}_{C}([t, \infty) \mid x)$ in (3.1), on the support of $H_{1}(\cdot \mid$ $x)$, uniformly with respect to $x$. A usual way to deal with this technical difficulty is to consider a finite threshold $\tau(x)$, that could depend on the covariate vector value $x$, beyond which no uncensored lifetime is observed, i.e., for each $x$,

$$
\tau(x) \stackrel{\text { def }}{=} \tau_{H_{1}}(x)=\inf _{t}\left\{H_{1}((t, \infty) \mid x)=0\right\}<\infty .
$$

Moreover, to be able to keep denominators, such as in equation (3.1), away from zero, we require the condition

$$
\inf _{x \in \mathcal{X}} H_{1}(\{\tau(x)\} \mid x) H_{0}((\tau(x), \infty) \mid x)>0 .
$$

In particular, this condition implies $\tau_{H_{0}}(x)>\tau(x), \forall x \in \mathcal{X}$. Moreover, given condition (2.3), necessarily $H_{0}(\{\tau(x)\} \mid x)=0, \forall x$. This means that $F_{C}(\{\tau(x)\} \mid x)=0, \forall x$. This constraint on $H_{0}(\{\tau(x)\} \mid x)$ could be relaxed at the expense of suitable adjustments of the inversion formulae. For simplicity, we keep condition (2.3). Let us also notice that condition (4.2) $\operatorname{implies}_{\inf } F_{C}((\tau(x), \infty) \mid x)>0$, and $\inf _{x} F_{T, 0}(\{\tau(x)\} \mid x)>0$.

Conditions like in equations (4.1)-(4.2) are more or less explicitly used in the literature of cure models, where so far the threshold $\tau$ was always considered independent of the covariate values. Sometimes $\tau$ is justified as 
representing a total follow-up of the study. For instance, Lu (2008) supposes that $Y=\min \{T, \min (C, \tau)\}$ and $\delta=\mathbf{1}\{T \leq \min (C, \tau)\}$, where $T=\eta T^{*}+$ $(1-\eta) \infty$, with $T^{*}<\infty$ and $\eta \in\{0,1\}$. The conditional probability of being cured is precisely the conditional probability of the event $\{\eta=0\}$. Next, Lu (2008) supposes that $\inf _{x} \mathbb{P}(\tau \leq T \leq C \mid x)>0$, and $\Lambda_{0}(\tau)<\infty$, where $\Lambda_{0}(\cdot)$ is the cumulative hazard function of $T^{*}$. All these conditions together clearly imply our conditions (4.1)-(4.2).

Fang et al. (2005) implicitly restrict the uncensored lifetimes to some compact interval $[0, \tau]$ and suppose $\mathbb{E}(\delta \mathbf{1}\{Y \geq \tau\})>0$. This could be possible only if $H_{1}(\{\tau\} \mid x)>0$ for a set of values $x$ with positive probability. In a proportional hazards context with the covariates taking values in a bounded set, as assumed by Fang et al. (2005), this is equivalent to $H_{1}(\{\tau\} \mid x) \geq c>0$ for almost all $x$, for some constant $c$.

The fact that technical conditions similar to our conditions (4.1)-(4.2) could be traced in the cure models literature is not unexpected in view of our Section 2.5. Indeed, the existing approaches could be interpreted through our inversion formulae and thus the technical problems we face in the asymptotic investigation are expected to be also present in the alternative approaches.

4.1. Consistency. Let us sketch the arguments we use for proving the consistency of $\widehat{\beta}$. On one hand, if the conditional subdistributions $H_{k}(\cdot \mid x)$ are given, one can build the purely parametric likelihood

$$
\mathcal{L}_{n}(\beta)=\prod_{i=1}^{n} \phi\left(X_{i}, \beta\right)^{\delta_{i}}\left\{\phi\left(X_{i}, \beta\right) F_{T, 0}\left(\left[Y_{i}, \infty\right) \mid X_{i}\right)+1-\phi\left(X_{i}, \beta\right)\right\}^{1-\delta_{i}}
$$

By construction, $\mathcal{L}_{n}(\beta)$ is a functional of $H_{0}(\cdot \mid x)$ and $H_{1}(\cdot \mid x), x \in \mathcal{X}$, while $\widehat{L}_{n}(\beta)$ is a functional of their estimated versions. Hence, for deriving the consistency of $\widehat{\beta}$, first we show that $\beta_{0}$ defined by equation (2.13) is a well-separated maximum of the function $\beta \mapsto \mathbb{E}\left[\log \mathcal{L}_{n}(\beta)\right]$ and that $\sup _{\beta \in B}\left|\log \mathcal{L}_{n}(\beta)-\mathbb{E}\left[\log \mathcal{L}_{n}(\beta)\right]\right|=o_{\mathbb{P}}(n)$.

Next, we check that

$$
\sup _{\beta \in B}\left|\log \widehat{L}_{n}(\beta)-\log \mathcal{L}_{n}(\beta)\right|=o_{\mathbb{P}}(n) .
$$

We then have that $\log \mathcal{L}_{n}(\widehat{\beta}) \geq \sup _{\beta \in B} \log \mathcal{L}_{n}(\beta)-o_{\mathbb{P}}(n)$. From this we will derive the consistency of $\widehat{\beta}$ using results from Section 5.2 in van der Vaart (1998). To prove condition (4.4), we have to guarantee the uniform convergence of $\widehat{H}_{k}-H_{k}, k \in\{0,1\}$, as stated in Assumption (AC1) below.

Formally, to prove the consistency of $\widehat{\beta}$, we use the following assumptions: 
(AC1) For $\tau(x)$ appearing in conditions (4.1)-(4.2),

$$
\sup _{x \in \mathcal{X}} \sup _{t \in(-\infty, \tau(x)]}\left|\widehat{H}_{k}([t, \infty) \mid x)-H_{k}([t, \infty) \mid x)\right|=o_{\mathbb{P}}(1), \quad k \in\{0,1\} .
$$

(AC2) The parameter set $B \subset \mathbb{R}^{p}$ is compact.

(AC3) There exist some constants $a>0$ and $c_{1}>0$ such that

$$
\left|\phi(x, \beta)-\phi\left(x, \beta^{\prime}\right)\right| \leq c_{1}\left\|\beta-\beta^{\prime}\right\|^{a}, \quad \forall \beta, \beta^{\prime} \in B, \forall x, x^{\prime} \in \mathcal{X},
$$

where $\|A\|$ is the Euclidean norm of any vector $A$.

$(\mathrm{AC} 4) \inf _{\beta \in B} \inf _{x \in \mathcal{X}} \phi(x, \beta)>0$.

Theorem 4.1. Assume that (AC1)-(AC4) and (2.2), (2.3), (2.8), (2.14), (4.1) and (4.2) hold true. Moreover, assume that there exists a unique value $\beta_{0}$ in the parameter set $B$ such that (2.13) is true. Then, $\widehat{\beta}-\beta_{0}=o_{\mathbb{P}}(1)$.

Let us point out that the consistency result is stated in terms of the subdistributions of the observations and the conditional probability model $\{\phi(\cdot, \beta): \beta \in B\}$. If the model is correctly specified, $\phi(x, \widehat{\beta})$ consistently estimates the cure probability $\mathbb{P}(T=\infty \mid x)$ for all $x$ in the support of $X$. Let us also notice that condition (AC3) guarantees the Glivenko-Cantelli property we use in the proof and it will be satisfied in the common modeling situations. Condition (AC4) is a weak condition on the model $\phi(x, \beta)$ and is e.g. satisfied for the logistic model if $\mathcal{X}$ and $B$ are compact.

4.2. Asymptotic normality. For the asymptotic normality we use the approach in Chen et al. (2003), Section 3.2. For this purpose we use the derivative of $\log \widehat{L}_{n}(\beta)$ with respect to $\beta$ and we embed the nuisance functions $H_{k}([\cdot, \infty) \mid \cdot)(k=0,1)$ in a functional space $\mathcal{H}$ equipped with a norm $\|\cdot\|_{\mathcal{H}} \cdot$ Both the space $\mathcal{H}$ and its norm $\|\cdot\|_{\mathcal{H}}$ will be chosen depending on the estimators $\widehat{H}_{k}([\cdot, \infty) \mid \cdot), k=0,1$, and have to satisfy certain conditions, given below. The true vector of nuisance functions is

$$
\eta_{0}(t, x)=\left(\eta_{01}(t, x), \eta_{02}(t, x)\right)=\left(H_{0}([t, \infty) \mid x), H_{1}([t, \infty) \mid x)\right) .
$$

For each $x \in \mathcal{X}$ and for each $\eta_{1}, \eta_{2} \in \mathcal{H}$, let $\eta(d t, x)=\left(\eta_{1}(d t, x), \eta_{2}(d t, x)\right)$ be the measures associated to the functions $\eta(\cdot, x)=\left(\eta_{1}(\cdot, x), \eta_{2}(\cdot, x)\right)$.

Let $\nabla_{\beta}$ denote the vector-valued partial differentiation operator with respect to the components of $\beta$. First, note that the vector of partial derivatives of the $\log$-likelihood $\log \widehat{L}_{n}(\beta)$ with respect to the components of $\beta$ equals

$$
\nabla_{\beta} \log \widehat{L}_{n}(\beta)=\frac{1}{n} \sum_{i=1}^{n} m\left(Y_{i}, \delta_{i}, X_{i} ; \beta, \widehat{\eta}\right), \quad \widehat{\eta}=\left(\widehat{H}_{0}, \widehat{H}_{1}\right),
$$


and

$$
m(t, d, x ; \beta, \eta)=\left\{\frac{d}{\phi(x, \beta)}-\frac{(1-d) T(\eta)(t, x)}{1-\phi(x, \beta) T(\eta)(t, x)}\right\} \nabla_{\beta} \phi(x, \beta) .
$$

The map $\eta \mapsto T(\eta)(\cdot, \cdot)$ is given by

$$
T(\eta)(t, x)=F_{T, 0}((-\infty, t] \mid x)
$$

and defines $F_{T, 0}$ as a functional of $H_{0}$ and $H_{1}$ via the composition

$$
\left(H_{0}, H_{1}\right)(\cdot \mid \cdot) \mapsto\left(\mathbb{P}(T=\infty \mid \cdot),\left(F_{C}, H_{0}, H_{1}\right)(\cdot \mid \cdot)\right) \mapsto \Lambda_{T, 0}(\cdot \mid \cdot) \mapsto F_{T, 0}(\cdot \mid \cdot) .
$$

The maps in the above composition are defined in equations (2.7)-(2.5)-(2.6), (2.11) and (2.12), respectively. We also define

$$
M_{n}(\beta, \eta)=\frac{1}{n} \sum_{i=1}^{n} m\left(Y_{i}, \delta_{i}, X_{i} ; \beta, \eta\right) \quad \text { and } \quad M(\beta, \eta)=\mathbb{E}[m(Y, \delta, X ; \beta, \eta)] .
$$

Hence, we have that $M\left(\beta_{0}, \eta_{0}\right)=0$ and $\left\|M_{n}(\widehat{\beta}, \widehat{\eta})\right\|=\inf _{\beta \in B}\left\|M_{n}(\beta, \widehat{\eta})\right\|$, where $\widehat{\eta}(t, x)=\left(\widehat{\eta}_{1}(t, x), \widehat{\eta}_{2}(t, x)\right)=\left(\widehat{H}_{0}([t, \infty) \mid x), \widehat{H}_{1}([t, \infty) \mid x)\right)$.

Next, we have to investigate the derivatives of $M(\beta, \eta)$ with respect to $\beta$ and $\eta$. We will show in the proof of Theorem 4.2 below that

$$
\nabla_{\beta} M\left(\beta_{0}, \eta_{0}\right)=-\mathbb{E}\left\{W(X) \nabla_{\beta} \phi\left(X, \beta_{0}\right) \nabla_{\beta} \phi\left(X, \beta_{0}\right)^{\top}\right\}
$$

with the positive function $W(\cdot)$ given by

$$
\begin{aligned}
& W(x)=\frac{\int_{(-\infty, \tau(x)]} F_{C}((t, \infty) \mid x) F_{T, 0}(d t \mid x)}{\mathbb{P}(T}<\infty \mid x) \\
& \quad+\int_{(-\infty, \tau(x)]} \frac{F_{T, 0}^{2}((-\infty, t] \mid x) F_{C}(d t \mid x)}{1-\mathbb{P}(T<\infty \mid x) F_{T, 0}((-\infty, t] \mid x)} .
\end{aligned}
$$

Further, define the Gâteaux derivative of $M\left(\beta, \eta_{0}\right)$ in the direction $\left[\eta-\eta_{0}\right]$

$$
\nabla_{\eta} M\left(\beta, \eta_{0}\right)\left[\eta-\eta_{0}\right]=\lim _{\tau \rightarrow 0} \frac{1}{\tau}\left[M\left(\beta, \eta_{0}+\tau\left(\eta-\eta_{0}\right)\right)-M\left(\beta, \eta_{0}\right)\right] .
$$

We show in the proof of Theorem 4.2 below how to compute this derivative.

We make the following assumptions:

(AN1) The value $\beta_{0}$ is an interior point of the compact set $B \subset \mathbb{R}^{p}$. For any $x \in \mathcal{X}$, the function $\beta \rightarrow \phi(x, \beta), \beta \in B$, is twice continuously differentiable and the first and second order partial derivatives are bounded uniformly in $(x, \beta) \in \mathcal{X} \times B$. 
$(\mathrm{AN} 2) H_{k}([\cdot, \infty) \mid \cdot) \in \mathcal{H}$ for $k=0,1$

(AN3) The matrix $\mathbb{E}\left\{\nabla_{\beta} \phi\left(X, \beta_{0}\right) \nabla_{\beta} \phi\left(X, \beta_{0}\right)^{\top}\right\}$ is positive definite.

(AN4) For $k=0,1$, the estimator $\widehat{H}_{k}([\cdot, \infty) \mid \cdot)$ satisfies the following :

(i) $\mathbb{P}\left(\widehat{H}_{k}([\cdot, \infty) \mid \cdot) \in \mathcal{H}\right) \rightarrow 1$;

(ii) $\left\|\left(\widehat{H}_{k}-H_{k}\right)([\cdot, \infty) \mid \cdot)\right\|_{\mathcal{H}}=o_{\mathbb{P}}\left(n^{-1 / 4}\right)$;

(iii) For some functions $\psi_{j k}, j=1, \ldots 5, k=0,1$, defined in (8.3) in the Appendix, there exist functions $\Psi_{1}$ and $\Psi_{2}$, such that

$$
\begin{array}{r}
\sum_{k=0}^{1} \mathbb{E}^{*}\left[\psi_{1 k}(Y, X) \int_{(-\infty, Y)} \psi_{2 k}(u, X) d\left(\left(\widehat{H}_{k}-H_{k}\right)([u, \infty) \mid X)\right)\right] \\
=\frac{1}{n} \sum_{i=1}^{n} \Psi_{1}\left(Y_{i}, \delta_{i}, X_{i}\right)+R_{1 n}
\end{array}
$$

and

$$
\begin{array}{r}
\sum_{k, \ell=0}^{1} \mathbb{E}^{*}\left[\psi_{3 k}(Y, X) \int_{(-\infty, Y)} \psi_{4 k}(u, X) \psi_{5 k}\left(\left(\widehat{H}_{k}-H_{k}\right)([u, \infty) \mid X), X\right)\right. \\
\left.\times d H_{\ell}(u \mid X)\right]=\frac{1}{n} \sum_{i=1}^{n} \Psi_{2}\left(Y_{i}, \delta_{i}, X_{i}\right)+R_{2 n},
\end{array}
$$

where $\mathbb{E}^{*}$ denotes conditional expectation given the sample, taken with respect to the generic variables $Y, \delta, X$. Moreover,

$$
\mathbb{E}\left[\Psi_{1}(Y, \delta, X)\right]=\mathbb{E}\left[\Psi_{2}(Y, \delta, X)\right]=0, \quad\left\|R_{1 n}\right\|+\left\|R_{2 n}\right\|=o_{\mathbb{P}}\left(n^{-1 / 2}\right) .
$$

(AN5) The class $\mathcal{H}$ satisfies $\int_{0}^{\infty} \sqrt{\log N\left(\varepsilon, \mathcal{H},\|\cdot\|_{\mathcal{H}}\right)} d \varepsilon<\infty$, where $N(\varepsilon, \mathcal{H}, \| \cdot$ $\left.\|_{\mathcal{H}}\right)$ is the $\varepsilon$-covering number of the space $\mathcal{H}$ with respect to $\|\cdot\|_{\mathcal{H}}$.

Theorem 4.2. Assume that $\widehat{\beta}-\beta_{0}=o_{\mathbb{P}}(1)$ and that (AN1)-(AN5) and (2.2), (2.3), (2.8), (2.14), (4.1) and (4.2) hold true. Then,

$$
n^{1 / 2}\left(\widehat{\beta}-\beta_{0}\right) \Rightarrow \mathcal{N}\left(0,\left\{\nabla_{\beta} M\left(\beta_{0}, \eta_{0}\right)\right\}^{-1} V\left\{\nabla_{\beta} M\left(\beta_{0}, \eta_{0}\right)\right\}^{-1}\right),
$$

where $V=\operatorname{Var}\left(m\left(Y, \delta, X ; \beta_{0}, \eta_{0}\right)+\Psi_{1}(Y, \delta, X)+\Psi_{2}(Y, \delta, X)\right), \nabla_{\beta} M\left(\beta_{0}, \eta_{0}\right)$ is given in (4.7), and $\Psi_{1}$ and $\Psi_{2}$ are defined in Assumption (AN4)(iii).

The structure of the asymptotic variance of $\widehat{\beta}$ reveals the differences with a standard binary outcome regression. The expression of the functions $\nabla_{\beta} M$ and $m$ include terms depending on $H_{0}$ and $H_{1}$ which account for the uncertainty on the censored observations, which could correspond to cured or uncured subjects. The expression of the variance $V$ includes the functions $\Psi_{1}$ and $\Psi_{2}$ which account for the fact that $H_{0}$ and $H_{1}$ are unknown. 
4.3. Bootstrap consistency. Although in principle one can use Theorem 4.2 above for making inference, the asymptotic variance of $\widehat{\beta}$ has a complicated structure, and estimating it would not only be cumbersome, but the precision of the estimate for small samples could moreover be rather poor. In this section we show that a bootstrap procedure can be used to estimate the asymptotic variance of $\widehat{\beta}$, to approximate the whole distribution of $\widehat{\beta}$ or to construct confidence intervals or test hypotheses regarding $\beta_{0}$.

Here, we propose to use a naive bootstrap procedure, consisting in drawing triplets $\left(Y_{i}^{*}, \delta_{i}^{*}, X_{i}^{*}\right), 1 \leq i \leq n$, randomly with replacement from the data $\left(Y_{i}, \delta_{i}, X_{i}\right), 1 \leq i \leq n$. Let $\widehat{H}_{k}^{*}$ be the same estimator as $\widehat{H}_{k}(k=$ $0,1)$ but based on the bootstrap data, and for each $(\beta, \eta)$ let $M_{n}^{*}(\beta, \eta)=$ $n^{-1} \sum_{i=1}^{n} m\left(Y_{i}^{*}, \delta_{i}^{*}, X_{i}^{*} ; \beta, \eta\right)$. Define the bootstrap estimator $\widehat{\beta}^{*}$ to be any sequence satisfying $\left\|M_{n}^{*}\left(\widehat{\beta}^{*}, \widehat{\eta}^{*}\right)-M_{n}(\widehat{\beta}, \widehat{\eta})\right\|=\inf _{\beta \in B}\left\|M_{n}^{*}\left(\beta, \widehat{\eta}^{*}\right)-M_{n}(\widehat{\beta}, \widehat{\eta})\right\|$, where $\widehat{\eta}^{*}(t, x)=\left(\widehat{\eta}_{1}^{*}(t, x), \widehat{\eta}_{2}^{*}(t, x)\right)=\left(\widehat{H}_{0}^{*}([t, \infty) \mid x), \widehat{H}_{1}^{*}([t, \infty) \mid x)\right)$.

The following result, proved in the Supplement, shows that the bootstrap works, in the sense that it allows to recover correctly the distribution of $n^{1 / 2}\left(\widehat{\beta}-\beta_{0}\right)$.

TheOREM 4.3. Assume that $\widehat{\beta}-\beta_{0}=o_{\mathbb{P}}(1)$ and that (AN1)-(AN5) hold true. Moreover, assume that $\nabla_{\beta} M(\beta, \eta)$ is continuous in $\eta$ (with respect to $\left.\|\cdot\|_{\mathcal{H}}\right)$ at $(\beta, \eta)=\left(\beta_{0}, \eta_{0}\right)$, and that $\left(A N_{4}\right)$ holds true with $\widehat{H}_{k}-H_{k}$ replaced by $\widehat{H}_{k}^{*}-\widehat{H}_{k}(k=0,1)$ in $\mathbb{P}^{*}$-probability. Then,

$$
\sup _{u \in \mathbb{R}^{p}}\left|\mathbb{P}^{*}\left(n^{1 / 2}\left(\widehat{\beta}^{*}-\widehat{\beta}\right) \leq u\right)-\mathbb{P}\left(n^{1 / 2}\left(\widehat{\beta}-\beta_{0}\right) \leq u\right)\right|=o_{\mathbb{P}}(1),
$$

where $\mathbb{P}^{*}$ denotes probability conditionally on the data, and where the inequality sign means the component-wise inequality for vectors.

4.4. Verification of the assumptions for kernel estimators. Next, we illustrate the verification of the assumptions of our asymptotic results when the conditional subdistributions $H_{k}$ are estimated by means of kernel smoothing.

Consider the case where $X$ is composed of continuous and discrete components, that is $X=\left(X_{c}, X_{d}\right) \in \mathcal{X}_{c} \times \mathcal{X}_{d} \subset \mathbb{R}^{d_{c}} \times \mathbb{R}^{d_{d}}$, with $d_{c}+d_{d}=d \geq 1$. For simplicity, assume that the support $\mathcal{X}_{d}$ of the discrete subvector $X_{d}$ is finite. We also assume that the lifetime $T$ has not been transformed by a logarithmic or other transformation, so that its support is $(0, \infty]$. The subdistributions $H_{k}([t, \infty) \mid x)$ are assumed continuous in both arguments $t$ and $x$ and are estimated by means of a kernel estimator:

$$
\widehat{H}_{k}([t, \infty) \mid x)=\sum_{i=1}^{n} \frac{\widetilde{K}_{h_{n}}\left(X_{i}-x\right)}{\sum_{j=1}^{n} \widetilde{K}_{h_{n}}\left(X_{j}-x\right)} I\left(Y_{i} \geq t, \delta_{i}=k\right),
$$


where for any $\left(x_{c}, x_{d}\right) \in \mathcal{X}_{c} \times \mathcal{X}_{d}, \widetilde{K}_{h_{n}}\left(X_{i}-x\right)=K_{h_{n}}\left(X_{c, i}-x_{c}\right) I\left(X_{d, i}=x_{d}\right)$, $h_{n}$ is a bandwidth sequence, $K_{h}(\cdot)=K(\cdot / h) / h^{d_{c}}, K(u)=\prod_{j=1}^{d_{c}} k\left(u_{j}\right)$ and $k(\cdot)$ is a probability density function.

Nonparametric smoothing of continuous covariates is possible for dimensions $d_{c}$ larger than 1 . However, the technical arguments necessary to verify the assumptions used for the asymptotic results are tedious. Therefore, in the following we consider $d_{c}=1$. The discrete covariates do not contribute to the curse of dimensionality, and therefore $d_{d}$ could be larger than 1 . However, for simplicity, below we do not consider discrete covariates.

To satisfy assumption (AN4), we impose the following conditions:

(C1) The sequence $h_{n}$ satisfies $n h_{n}^{4} \rightarrow 0$ and $n h_{n}^{3+\zeta} / \log n \rightarrow \infty$ for some $\zeta>0$.

(C2) The support $\mathcal{X}$ of $X$ is a compact subset of $\mathbb{R}$ and the density $f_{X}(\cdot)$ of $X$ is bounded and bounded away from zero.

(C3) The probability density function $K$ has compact support, $\int u K(u) d u=$ 0 and $K$ is twice continuously differentiable.

(C4) There exists $\tau$ such that $\sup _{x} \tau(x)<\tau<\inf _{x} \tau_{H_{0}}(x) \leq \infty$.

Further, let $\mathcal{F}_{1}$ be the space of functions from $[0, \tau]$ to $[0,1]$ with variation bounded by $0<M<\infty$, and let $\mathcal{F}_{2}$ be the space of continuously differentiable functions $f$ from $\mathcal{X}$ to $[-M, M]$ that satisfy $\sup _{x \in \mathcal{X}}\left|f^{\prime}(x)\right| \leq M$ and $\sup _{x_{1}, x_{2} \in \mathcal{X}}\left|f^{\prime}\left(x_{1}\right)-f^{\prime}\left(x_{2}\right)\right| /\left|x_{1}-x_{2}\right|^{\epsilon} \leq M$ for some $0<\epsilon<1$. Let

$$
\begin{gathered}
\mathcal{H}=\left\{(t, x) \rightarrow \eta(t, x): \eta(\cdot, x) \in \mathcal{F}_{1}, \frac{\partial}{\partial x} \eta(\cdot, x) \in \mathcal{F}_{1} \text { for all } x \in \mathcal{X},\right. \\
\text { and } \left.\eta(t, \cdot) \in \mathcal{F}_{2} \text { for all } 0 \leq t \leq \tau\right\}
\end{gathered}
$$

We define the following norm associated with the space $\mathcal{H}$ : for $\eta \in \mathcal{H}$, let $\|\eta\|_{\mathcal{H}}=\sup _{0 \leq t \leq \tau} \sup _{x \in \mathcal{X}}|\eta(t, x)|$. Then, it follows from Propositions 1 and 2 in Akritas and Van Keilegom (2001) that $\mathbb{P}\left(\widehat{H}_{k} \in \mathcal{H}\right) \rightarrow 1$ provided $n h_{n}^{3+\zeta}(\log n)^{-1} \rightarrow \infty$, with $\zeta>0$ as in condition (C1). Moreover, $\sup _{x, t}\left|\widehat{H}_{k}([t, \infty) \mid x)-H_{k}([t, \infty) \mid x)\right|=O_{\mathbb{P}}\left(\left(n h_{n}\right)^{-1 / 2}(\log n)^{1 / 2}\right)=o_{\mathbb{P}}\left(n^{-1 / 4}\right)$ (see Proposition 1 in Akritas and Van Keilegom 2001). The class $\mathcal{H}$ satisfies assumption (AN5) thanks to Lemma 6.1 in Lopez (2011). It remains to show the validity of assumption (AN4) ( $i i i)$. We will show the first statement, the second one can be shown in a similar way. Note that the left hand side equals

$$
\begin{aligned}
& \sum_{k=0}^{1} \mathbb{E}_{i}\left[\psi_{1 k}(Y, X) \int_{(0, Y)} \psi_{2 k}(u, X) \frac{1}{n} f_{X}^{-1}(X)\right. \\
& \left.\quad \times \sum_{i=1}^{n} K_{h_{n}}\left(X_{i}-X\right) d\left(I\left(Y_{i} \geq u, \delta_{i}=k\right)-H_{k}([u, \infty) \mid X)\right)\right]+o_{\mathbb{P}}\left(n^{-1 / 2}\right)
\end{aligned}
$$




$$
\begin{aligned}
= & \sum_{k=0}^{1} \mathbb{E}_{i}\left[\frac { \psi _ { 1 k } ( Y , X ) } { f _ { X } ( X ) } \frac { 1 } { n } \sum _ { i = 1 } ^ { n } K _ { h _ { n } } ( X _ { i } - X ) \left\{-\psi_{2 k}\left(Y_{i}, X\right) I\left(Y_{i} \leq Y, \delta_{i}=k\right)\right.\right. \\
& \left.\left.-\int_{(0, Y)} \psi_{2 k}(u, X) d H_{k}([u, \infty) \mid X)\right\}\right]+o_{\mathbb{P}}\left(n^{-1 / 2}\right) \\
= & \frac{1}{n} \Psi_{1}\left(Y_{i}, \delta_{i}, X_{i}\right)+o_{\mathbb{P}}\left(n^{-1 / 2}+h_{n}^{2}\right),
\end{aligned}
$$

where $\mathbb{E}_{i}[\cdot]=\mathbb{E}\left[\cdot \mid Y_{i}, \delta_{i}, X_{i}\right], \mathbb{E}_{i}\left[\cdot \mid X=X_{i}\right]=\mathbb{E}\left[\cdot \mid Y_{i}, \delta_{i}, X_{i}, X=X_{i}\right]$ and

$$
\begin{aligned}
& \Psi_{1}\left(Y_{i}, \delta_{i}, X_{i}\right)=-\sum_{k=0}^{1}\left\{\mathbb{E}_{i}\left(\psi_{1 k}\left(Y, X_{i}\right) I\left(Y_{i} \leq Y, \delta_{i}=k\right) \mid X=X_{i}\right) \psi_{2 k}\left(Y_{i}, X_{i}\right)\right. \\
& \left.-\int \mathbb{E}_{i}\left(\psi_{1 k}\left(Y, X_{i}\right) I\left(u \leq Y, \delta_{i}=k\right) \mid X=X_{i}\right) \psi_{2 k}\left(u, X_{i}\right) d H\left((-\infty, u] \mid X_{i}\right)\right\} .
\end{aligned}
$$

which is of the required form. Moreover, the $o_{\mathbb{P}}(\cdot)$ rates hold uniformly with respect to $i$, and we have $\mathbb{E}\left[\Psi_{1}\left(Y_{i}, \delta_{i}, X_{i}\right)\right]=0$.

Plugging the estimators $\widehat{H}_{0}, \widehat{H}_{1}$ and $\widehat{\beta}$ into the equations (2.11) and (2.12), we obtain an estimator for $F_{T, 0}$ which we denote by $\widetilde{F}_{T, 0}((t, \infty) \mid x)$. Then, under the additional conditions $(\mathrm{C} 1)$ to $(\mathrm{C} 4)$, with $\mathbb{P}(T \widehat{=\infty} \mid x)$ defined in equation (3.3), uniformly in $0<t \leq \tau$ and $x \in \mathcal{X}$, we have the following i.i.d. representation:

$$
\begin{aligned}
\widetilde{F}_{T, 0}((0, t] \mid x)-F_{T, 0} & ((0, t] \mid x) \\
& =\frac{1+o_{\mathbb{P}}(1)}{\phi\left(x, \beta_{0}\right) f_{X}(x)} \frac{1}{n} \sum_{i=1}^{n} K_{h_{n}}\left(h_{n}^{-1}\left(x-X_{i}\right)\right) \Upsilon_{i t},
\end{aligned}
$$

uniformly in $x$ and $t$, where

$$
\begin{aligned}
\Upsilon_{i t} & =\left\{1-\phi\left(x, \beta_{0}\right) F_{T, 0}((0, t] \mid x)\right\} \xi_{i t} \\
- & {\left[F_{T, 0}((0, t] \mid x)+\int_{(0, t]} \frac{\phi\left(x, \beta_{0}\right) F_{T, 0}((t, \tau] \mid x) F_{C}([s, \infty) \mid x) H_{1}(d s \mid x)}{\left\{H([s, \infty) \mid x)-\left[1-\phi\left(x, \beta_{0}\right)\right] F_{C}([s, \infty) \mid x)\right\}^{2}}\right] } \\
\times\left\{1-\phi\left(x, \beta_{0}\right)\right\} \xi_{i \tau} & \times\{1-\infty
\end{aligned}
$$

and

$$
\xi_{i t}=\frac{\delta_{i} I\left(Y_{i} \leq t\right)}{H\left(\left[Y_{i}, \infty\right) \mid x\right)}-\int_{\left(0, t \wedge Y_{i}\right]} \frac{H_{1}(d s \mid x)}{H^{2}([s, \infty) \mid x)}, \quad t \leq \tau .
$$

Par construction, $\Upsilon_{i \tau}=0$ and $\mathbb{E}\left[\Upsilon_{i t} \mid X_{i}=x\right]=0$. As a consequence of the i.i.d representation (4.9), weak convergence theorems for $\sqrt{n h_{n}}\left(\widetilde{F}_{T, 0}-F_{T, 0}\right)$ could be derived by standard arguments, that we omit for the sake of brevity. 
5. Efficiency aspects. Our modeling situation belongs to the general information loss model framework, see van der Vaart (1998), section 25.5.2. Here the observed variables $(Y, \delta, X)$ are a measurable transformation of the (partially) unobservable variables $(T, C, X)$. In order to separate the parametric and nonparametric parts of our model, we restate it using latent variables. Let $T_{0} \in \mathbb{R}$ be a latent finite lifetime and $\Delta$ be a latent binary variable such that $T=T_{0}$ when $\Delta=1$ and $T=\infty$ when $\Delta=0$. Thus we have $\{T<\infty\}=\{\Delta=1\}$. To account for the equations (2.1) and (2.2), we assume $\Delta \perp\left(T_{0}, C\right) \mid X$ and $T_{0} \perp C \mid X$. The infinite dimensional parameters of the model are given by the laws of $C, X$ and $T_{0}$. Given the structure of the likelihood, and assuming that the laws of $C$ and $X$ do not carry any information on the parameter of interest $\beta$, we could only consider submodels in the direction of $F_{T, 0}$. Then the score function for the path induced in our model by a submodel in the direction of $F_{T, 0}$, with score function $b(\cdot)$, is given by

$$
\begin{aligned}
g(Y, \delta, X) & =\mathbb{E}\left[b\left(T_{0}, X\right) \mid Y, \delta, X\right] \\
= & \delta b(Y, X)+(1-\delta) \phi(X, \beta) \frac{\int_{(Y, \infty)} b(t, X) d F_{T, 0}(t \mid X)}{1-\phi(X, \beta) F_{T, 0}((-\infty, Y] \mid X)}
\end{aligned}
$$

The tangent set, say $\mathcal{T}$, in the nonparametric direction of $F_{T, 0}$ is composed of vector-valued functions $g(\cdot)$ depending on the observations and defined by squared norm integrable centered vector-valued functions $b\left(T_{0}, X\right)$. To project the score of the parametric part of our model

$$
m_{0}(Y, \delta, X)=\left\{\frac{\delta}{\phi\left(X, \beta_{0}\right)}-\frac{(1-\delta) F_{T, 0}((-\infty, Y] \mid X)}{1-\phi\left(X, \beta_{0}\right) F_{T, 0}((-\infty, Y] \mid X)}\right\} \nabla_{\beta} \phi\left(X, \beta_{0}\right)
$$

onto the tangent set $\mathcal{T}$, we have to find a function $b_{0}\left(T_{0}, X\right)$, yielding $g_{0}(Y, \delta, X)$ defined as in equation (5.1), such that

$$
\mathbb{E}\left[\left\{m_{0}(Y, \delta, X)-g_{0}(Y, \delta, X)\right\} g_{*}(Y, \delta, X)\right]=0
$$

for all $g_{*}(Y, \delta, X)$ defined by score functions $b_{*}\left(T_{0}, X\right)$. In the Supplementary Material, we show that this equation is an integral equation with a solution that could be approximated numerically.

With this solution we then obtain the efficient score $S_{\text {eff }}\left(Y, \delta, X ; \beta_{0}, \eta_{0}\right)=$ $m_{0}(Y, \delta, X)-g_{0}(Y, \delta, X)$. A semi-parametrically efficient estimator could be obtained by solving the equation

$$
n^{-1} \sum_{i=1}^{n} S_{\mathrm{eff}}\left(Y_{i}, \delta_{i}, X_{i} ; \beta, \widehat{\eta}\right)=o_{\mathbb{P}}\left(n^{-1 / 2}\right)
$$


for $\beta$. The solution $\widehat{\beta}_{\text {eff }}$ will have the following i.i.d. representation:

$$
\widehat{\beta}_{\mathrm{eff}}-\beta_{0}=n^{-1} \sum_{i=1}^{n}\left[\mathbb{E}\left(S_{\mathrm{eff}, i} S_{\mathrm{eff}, i}^{\top}\right)\right]^{-1} S_{\mathrm{eff}, i}+o_{\mathbb{P}}\left(n^{-1 / 2}\right)
$$

where $S_{\text {eff }, i}=S_{\text {eff }}\left(Y_{i}, \delta_{i}, X_{i} ; \beta_{0}, \eta_{0}\right)$.

6. Simulations. In this section we will investigate the small sample performance of our estimation method. We consider the following model. The covariate $X$ is generated from a uniform distribution on $[-1,1]$, and the conditional probability $\phi(x, \beta)$ of not being cured follows a logistic model : $\phi(x, \beta)=\operatorname{logit}\left(\beta_{1}+\beta_{2} x\right)$, with $|x| \leq 1$. We will work with $\beta_{0}=$ $\left(\beta_{01}, \beta_{02}\right)=(1.75,2)$ and $(1.1,2)$, corresponding to an average cure rate of $20 \%$ respectively $30 \%$. The conditional distribution function $F_{T, 0}(\cdot \mid x)$ of the uncured individuals is constructed as follows. For a given $X=x$, we draw $T$ from a Weibull law: $F_{T, 0}([t, \infty) \mid x)=\exp \left(-t^{k(x)} \exp \left(\gamma_{0}+\gamma_{1} x\right)\right)$, where $k(x)=k+a(1 /(1+x)-1 / 2)$. We take $k=0.75, \gamma_{0}=0.5$ and $\gamma_{1}=1$, and $a \in\{0,1,2\}$. Next, in order to respect condition (4.2), we truncate this distribution at $\tau(x)$, where $\tau(x) \equiv \tau$ equals the quantile of order 0.97 of $F_{T, 0}(y \mid 0)$ when $a=0$, and the quantile of order 0.97 of $F_{T, 0}(y \mid x)$ when $a=1$ or 2 . Note that the Cox model is only verified if $a=0$, and in that case we have a baseline cumulative hazard function given by $\exp \left(\gamma_{0}\right) t^{k}$ for $t \leq \tau$ and given by infinity for $t>\tau$.

Next, we generate the censoring variable $C$ as follows: $F_{C}([t, \infty) \mid x)=$ $\exp \left(-t^{k(x)} \exp \left(\gamma_{0}+\gamma_{1} x\right) / p_{C}\right)$, where $p_{C}=\left(1-p_{\text {cens }}\right) /\left(p_{\text {cens }}-p_{\text {cure }}\right)$, with $p_{\text {cens }}$ the proportion of censored subjects in the population when $a=0$ and $p_{\text {cure }}$ the proportion of cured subjects in the population when $a=0$. When $p_{\text {cure }}=0.20$ we take $p_{\text {cens }}=0.25($ model 1$)$ and 0.35 (model 2$)$ and when $p_{\text {cure }}=0.30$ we take $p_{\text {cens }}=0.35($ model 3$)$ and $0.45($ model 4$)$.

In what follows we will compare our estimator of $\beta$ with the estimator proposed by $\mathrm{Lu}$ (2008) which assumes a Cox model for the uncured individuals. The estimated $\beta$ coefficients under the Cox model are obtained using the $\mathrm{R}$ package smcure. For our estimation procedure we used the kernel estimators given in Section 4.4, and we programmed $\widehat{\beta}$ using the optimization procedure optim in $\mathrm{R}$. The details of the maximization procedure we used are provided in the Supplementary Material. The results of our maximization procedure are given in Table 1 for the case where $\beta_{0}=(1.75,2)$ (model 1 and 2), and in Table 2 for the case where $\beta_{0}=(1.1,2)$ (model 3 and 4). A total of 1020 samples of size $n=200$ are generated, and the tables show the bias and the variance of the estimators $\widehat{\beta}_{1}$ and $\widehat{\beta}_{2}$ obtained under the Cox model and from our procedure. (Additional tables containing the 
results for the case $n=400$ are provided in the Supplement). For stability reasons, the results are truncated in the sense that the lowest and highest $1 \%$ of the estimators are omitted, and so the actual number of samples is 1000. The kernel function $K$ is taken equal to the Epanechnikov kernel : $K(u)=(3 / 4)\left(1-u^{2}\right) I(|u| \leq 1)$. For the bandwidth $h$ of the kernel estimators $\widehat{H}_{k}\left(\cdot \mid X_{i}\right)(k=0,1)$, defined as in (4.8), we used the cross-validation (CV) procedure proposed by Li et al. (2013) for kernel estimators of conditional distribution functions. The CV procedure is implemented in the package $\mathrm{np}$ in $\mathrm{R}$. For each sample in our simulation, we calculated these bandwidths for $\widehat{H}_{0}$ and $\widehat{H}_{1}$ and used the average of these two bandwidths, truncated to the interval $\left[n^{-2 / 7}, 5 n^{-2 / 7}\right]$ in order to verify regularity condition $(\mathrm{C} 1)$.

\begin{tabular}{|c|c|c|c|c|c|c|c|c|c|c|}
\hline \multirow[b]{3}{*}{$n$} & \multirow[b]{3}{*}{$a$} & \multirow[b]{3}{*}{ Par. } & \multicolumn{4}{|c|}{ Model 1} & \multicolumn{4}{|c|}{ Model 2} \\
\hline & & & \multicolumn{2}{|c|}{ New } & \multicolumn{2}{|c|}{ Cox } & \multicolumn{2}{|c|}{ New } & \multicolumn{2}{|c|}{ Cox } \\
\hline & & & Bias & Var & Bias & Var & Bias & Var & Bias & Var \\
\hline \multirow[t]{6}{*}{200} & 0 & $\beta_{1}$ & .010 & .059 & .044 & .063 & -.001 & .092 & .080 & .111 \\
\hline & & $\beta_{2}$ & -.034 & .175 & .061 & .186 & -.038 & .258 & .116 & .305 \\
\hline & 1 & $\beta_{1}$ & .025 & .058 & .063 & .053 & .032 & .088 & .087 & .082 \\
\hline & & $\beta_{2}$ & -.068 & .180 & -.350 & .175 & .093 & .273 & -.590 & .245 \\
\hline & 2 & $\beta_{1}$ & .027 & .058 & .093 & .053 & .030 & .079 & .085 & .074 \\
\hline & & $\beta_{2}$ & -.076 & .180 & -.489 & .172 & -.097 & .247 & -.593 & .220 \\
\hline
\end{tabular}

TABLE 1

Bias and Var of $\widehat{\beta}_{1}$ and $\widehat{\beta}_{2}$ for $n=200$ and for three values of $a$. Here, $\mathbb{P}($ cured $)=0.2$ and $\mathbb{P}$ (censoring) is either 0.25 (model 1) or 0.35 (model 2) for $a=0$. The Cox model is satisfied for $a=0$.

\begin{tabular}{|c|c|c|c|c|c|c|c|c|c|c|}
\hline \multirow[b]{3}{*}{$n$} & \multirow[b]{3}{*}{$a$} & \multirow[b]{3}{*}{ Par. } & \multicolumn{4}{|c|}{ Model 3} & \multicolumn{4}{|c|}{ Model 4} \\
\hline & & & \multicolumn{2}{|c|}{ New } & \multicolumn{2}{|c|}{ Cox } & \multicolumn{2}{|c|}{ New } & \multicolumn{2}{|c|}{ Cox } \\
\hline & & & Bias & Var & Bias & Var & Bias & Var & Bias & Var \\
\hline \multirow[t]{6}{*}{200} & 0 & $\beta_{1}$ & .002 & .039 & .024 & .040 & -.009 & .059 & .053 & .069 \\
\hline & & $\beta_{2}$ & -.039 & .136 & .039 & .138 & -.026 & .195 & .096 & .220 \\
\hline & 1 & $\beta_{1}$ & .017 & .038 & .057 & .036 & .017 & .059 & .076 & .058 \\
\hline & & $\beta_{2}$ & -.049 & .136 & -.255 & .140 & -.052 & .212 & -.427 & .198 \\
\hline & 2 & $\beta_{1}$ & .018 & .038 & .085 & .037 & .020 & .060 & .129 & .063 \\
\hline & & $\beta_{2}$ & -.052 & .136 & -.366 & .138 & -.056 & .214 & -.599 & .200 \\
\hline
\end{tabular}

TABLE 2

Bias and Var of $\widehat{\beta}_{1}$ and $\widehat{\beta}_{2}$ for $n=200$ and for three values of a. Here, $\mathbb{P}($ cured $)=0.3$ and $\mathbb{P}$ (censoring) is either 0.35 (model 3) or 0.45 (model 4 ) for $a=0$. The Cox model is satisfied for $a=0$.

The tables show that our estimator outperforms the one that is based on the Cox model when the Cox model is not verified, whereas when the model is satisfied (so when $a=0$ ) the two estimators behave rather similarly.

Next, we look at the estimation of the quartiles of the distribution $F_{T, 0}(\cdot \mid x)$ 

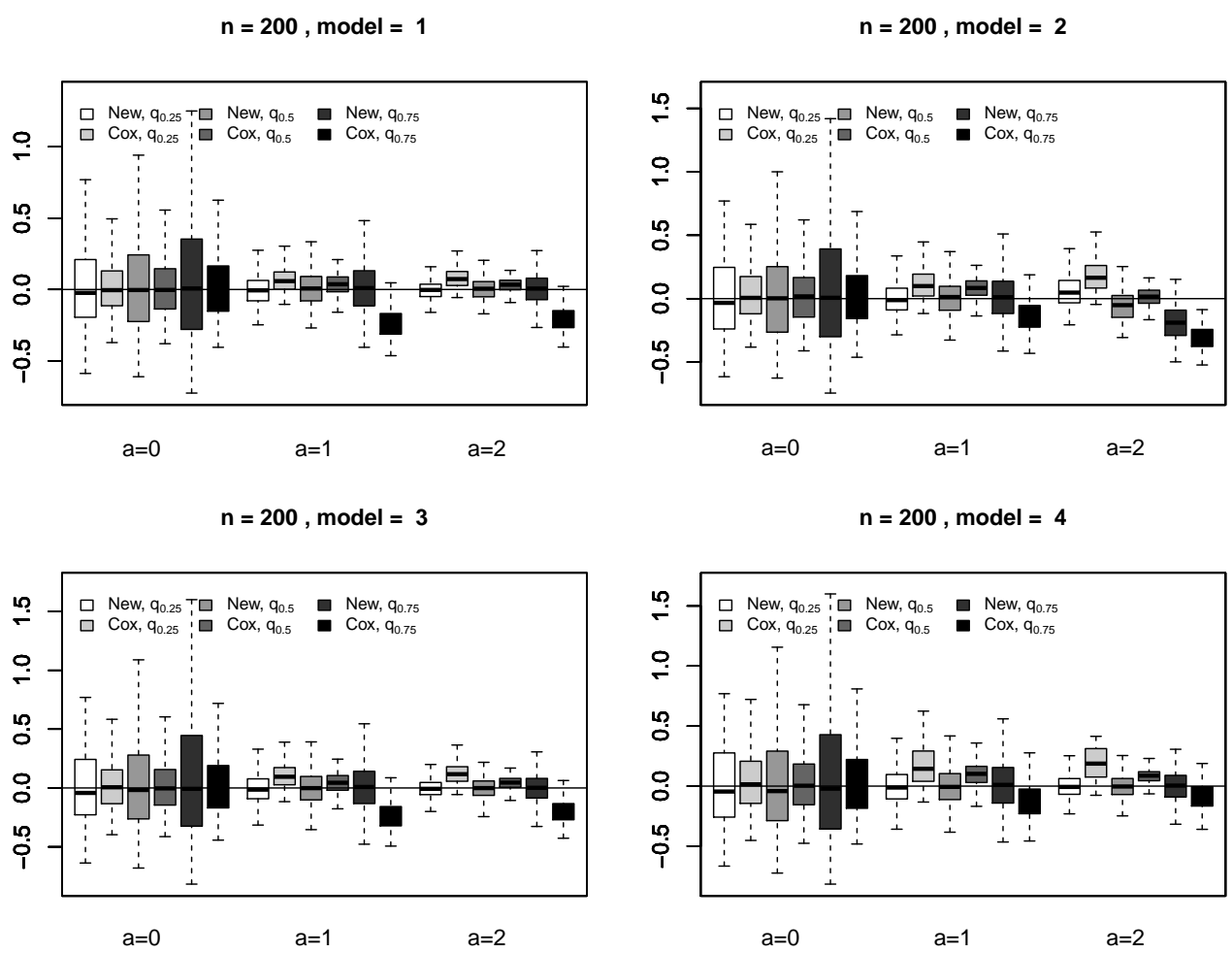

FIG 1. Boxplots for quantile estimators.

when $x=-0.5$. We estimate these quartiles by means of our nonparametric estimator $\widehat{F}_{T, 0}(\cdot \mid x)$ and by means of the Cox model studied in Lu (2008). The results given in Figure 1 for $n=200$ show that, as could be expected, when the Cox model is satisfied (i.e. when $a=0$ ) our quantile estimators have a larger variance than those obtained under the Cox model, whereas the bias is similar for both estimators. On the other hand, when the Cox model is not satisfied (i.e. when $a=1$ or 2), the estimated quartiles obtained under the Cox model are heavily biased. This shows the importance of having a model that does not impose any assumptions on the distribution of the uncured individuals and which still provides very accurate estimators for the logistic part of the model.

We also verify how close the distributions of $\widehat{\beta}_{1}$ and $\widehat{\beta}_{2}$ are to a normal distribution. We know thanks to Theorem 4.2 that the estimators converge to a normal limit when $n$ tends to infinity. In the Supplement we provide simulation results showing that the asymptotic normal approximation is already quite accurate for $n=200$. 
Next, we verify the accuracy of the naive bootstrap proposed in Section 4.3. Figure 2 shows boxplots of the variance of $\widehat{\beta}_{1}$ and $\widehat{\beta}_{2}$, centered around the empirical variance of the estimators of $\beta_{1}$ and $\beta_{2}$. The boxplots are obtained from 500 bootstrap resamples for each of 1000 samples of size $n=200$. The boxplots show that the bootstrap variance is well centered around the corresponding empirical variance.

We also obtain percentile bootstrap confidence intervals for $\beta_{1}$ and $\beta_{2}$, and calculate the coverage probability and average length of these intervals. The results are given in Table 3 and show that the coverage is very close to the desired $95 \%$ and that the results are better for $n=400$ than for $n=200$.

The objective of the next simulation study is twofold. First, we like to know how our method performs when the model depends on two covariates, and second we like to compare the performance of our method with that of the method proposed by Taylor (1995), which assumes that the latency does not depend on the covariates. The results given in the Supplement show that our method still performs well with two-dimensional smoothing when
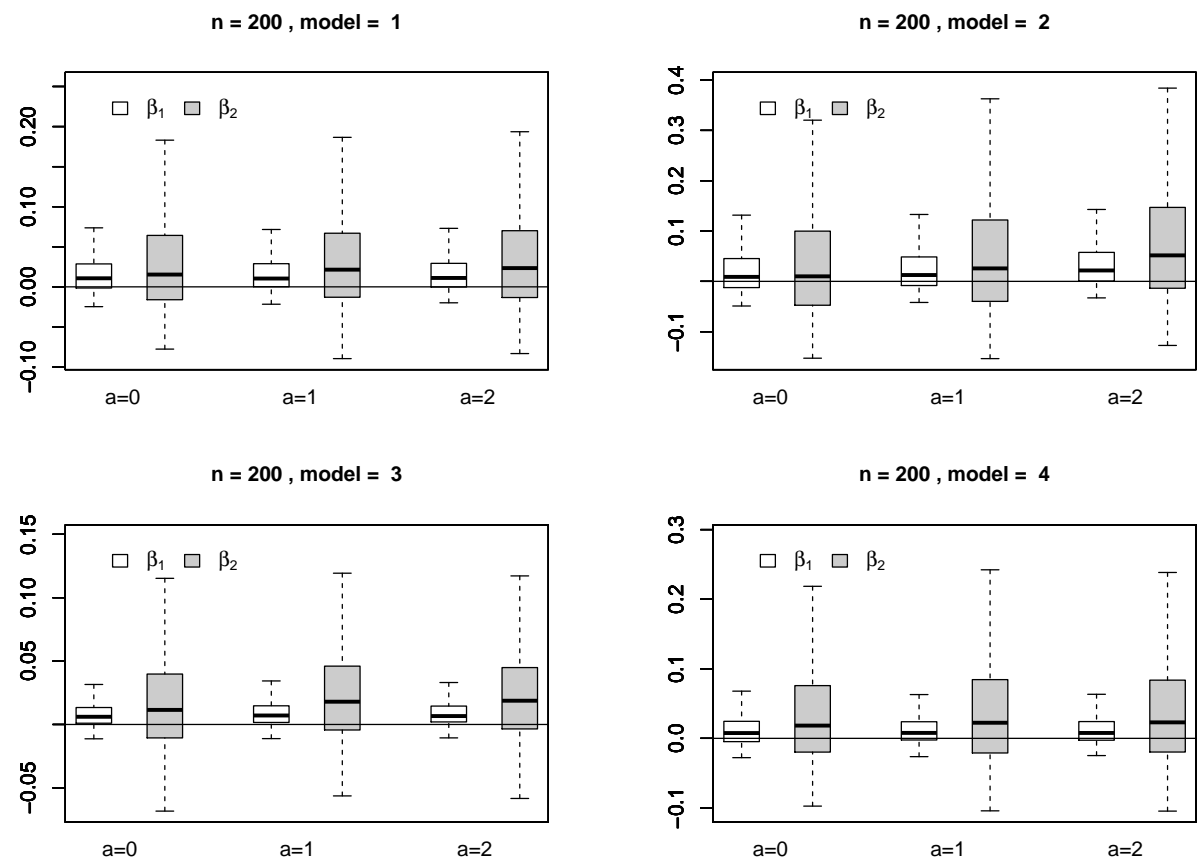

FIG 2. Boxplots of the bootstrap variance of $\widehat{\beta}_{1}$ and $\widehat{\beta}_{2}$, centered around the empirical variance of the estimators of $\beta_{1}$ and $\beta_{2}$. The boxplots are obtained from 500 bootstrap resamples for each of 1000 samples of size $n=200$, using the new estimation method. 


\begin{tabular}{|c|c|c|c|c|c|c|c|c|c|c|}
\hline \multirow[b]{3}{*}{$a$} & \multirow[b]{3}{*}{ Par. } & & \multicolumn{4}{|c|}{$n=200$} & \multicolumn{4}{|c|}{$n=400$} \\
\hline & & & \multicolumn{4}{|c|}{ Model } & \multicolumn{4}{|c|}{ Model } \\
\hline & & & 1 & 2 & 3 & 4 & 1 & 2 & 3 & 4 \\
\hline \multirow[t]{4}{*}{0} & $\beta_{1}$ & $\mathrm{Cov}$ & 0.97 & 0.96 & 0.96 & 0.96 & 0.95 & 0.96 & 0.95 & 0.96 \\
\hline & & Len & 1.07 & 1.31 & 0.85 & 1.05 & 0.73 & 0.90 & 0.58 & 0.73 \\
\hline & $\beta_{2}$ & Cov & 0.95 & 0.97 & 0.96 & 0.97 & 0.96 & 0.96 & 0.96 & 0.95 \\
\hline & & Len & 1.77 & 2.12 & 1.53 & 1.87 & 1.21 & 1.46 & 1.04 & 1.29 \\
\hline \multirow[t]{4}{*}{1} & $\beta_{1}$ & $\mathrm{Cov}$ & 0.97 & 0.96 & 0.95 & 0.96 & 0.95 & 0.96 & 0.95 & 0.97 \\
\hline & & Len & 1.07 & 1.31 & 0.85 & 1.05 & 0.73 & 0.89 & 0.58 & 0.73 \\
\hline & $\beta_{2}$ & Cov & 0.95 & 0.96 & 0.96 & 0.96 & 0.95 & 0.95 & 0.95 & 0.95 \\
\hline & & Len & 1.81 & 2.23 & 1.56 & 1.96 & 1.25 & 1.52 & 1.07 & 1.34 \\
\hline \multirow[t]{4}{*}{2} & $\beta_{1}$ & $\mathrm{Cov}$ & 0.97 & 0.97 & 0.95 & 0.96 & 0.95 & 0.96 & 0.95 & 0.96 \\
\hline & & Len & 1.07 & 1.31 & 0.85 & 1.05 & 0.73 & 0.89 & 0.58 & 0.73 \\
\hline & $\beta_{2}$ & Cov & 0.95 & 0.97 & 0.95 & 0.96 & 0.95 & 0.95 & 0.95 & 0.96 \\
\hline & & Len & 1.81 & 2.22 & 1.56 & 1.97 & 1.24 & 1.52 & 1.07 & 1.34 \\
\hline
\end{tabular}

TABLE 3

Coverage probability (Cov) and average length (Len) of bootstrap confidence intervals for $\widehat{\beta}_{1}$ and $\widehat{\beta}_{2}$ for two sample sizes and three values of $a$, using the new estimation method.

compared to the method under the Cox model, and that we outperform the method of Taylor when his model is not satisfied, whereas all three methods behave quite similarly when the three models are satisfied.

In the last simulation study, we investigate what happens when the model is misspecified. More precisely, we generate data from a model containing two covariates, and we want to know how our estimator and the estimator under the Cox model behave when fitting a mixture cure model containing only one of the two covariates. The results provided in the Supplement show that our nonparametric model on $F_{T, 0}$ is able to compensate better for the forgotten covariate. This is what is expected given that our model on $F_{T, 0}$ is nonparametric and hence more flexible than the Cox model.

7. Data analysis. Let us now apply our estimation procedure on a medical data set of 286 breast cancer patients with lymph-node-negative breast cancer treated between 1980 and 1995 (Wang et al. (2005)). The event of interest is distant-metastasis, and the associated survival time is the distant metastasis-free survival time (defined as the time to first distant progression or death, whichever comes first). 107 of the 286 patients experience a relapse from breast cancer. The plot of the Kaplan-Meier (KM) estimator of the data is given in Figure 3(a) and shows a large plateau at about 0.60 . Furthermore, a large proportion of the censored observations is in the plateau, which suggests that a cure model is appropriate for these data. As a covariate we use the age of the patients, which ranges from 26 to 
(a)

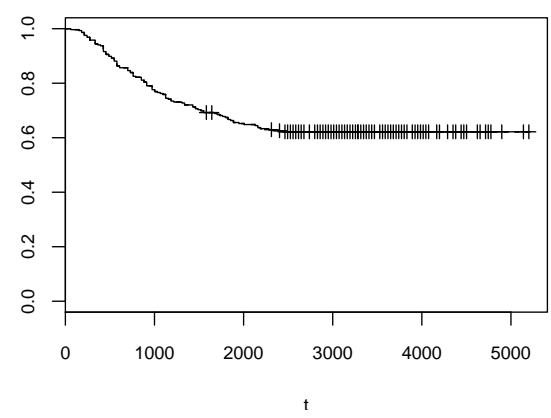

(c)

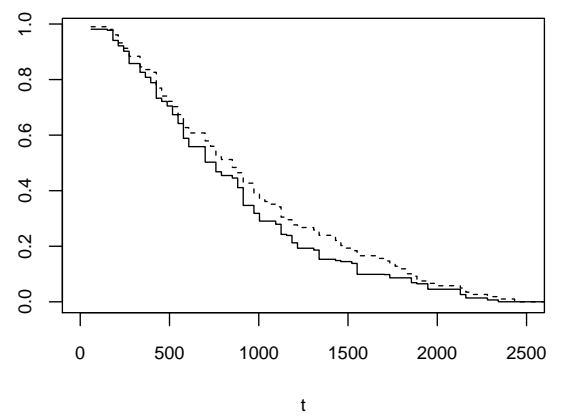

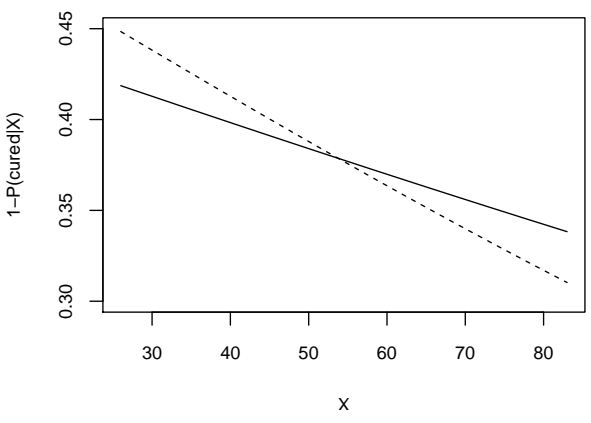

(d)

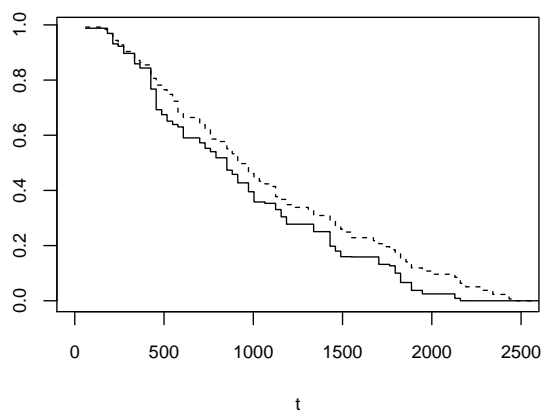

FIG 3. Analysis of the breast cancer data : (a) KM estimator; (b) Graph of the proposed estimator of $\phi(x)$ (solid curve) and of the estimator based on the Cox model (dashed curve); (c) Estimation of $1-F_{T, 0}(\cdot \mid x)$ using the proposed estimator (solid curve) and using the estimator based on the Cox model (dashed curve) when $x=48$; (d) Idem when $x=60$.

83 years and the average age is about 54 years.

We estimate $\beta$ using our estimator and using the estimator based on the Cox model. The bandwidth $h$ is selected using cross-validation, as in the simulation section. The estimated intercept is -0.168 (with standard deviation equal to 0.502 obtained using a naive bootstrap procedure), and the estimated slope parameter is -0.006 (with standard deviation equal to 0.009). Under the Cox model the estimated intercept and slope are respectively 0.063 and -0.010 . A $95 \%$ confidence interval is given by $(-1.152,0.815)$ for the intercept and $(-0.024,0.012)$ for the slope, where the variance is again based on the naive bootstrap procedure. The graph of the two estimators of the function $\phi(x)$ is given in Figure 3(b). The estimated coefficients and curves are quite close to each other, suggesting that the Cox model might be valid. This is also confirmed by Figure 3(c)-(d), which shows the estimation of the survival function of the uncured patients for $x=48$ and $x=60$ based 
on our estimation procedure and the one based on the Cox model. The figure shows that the two estimators are close for both values of $x$.

\section{Appendix: Technical lemmas and proofs.}

Lemma 8.1. Assume that conditions (2.2), (2.3) and (2.8) are met. Fix $x \in \mathcal{X}$ arbitrarily. Assume that $H_{1}(\cdot \mid x)$ puts a positive mass at the right endpoint of its right-bounded support. Then, $F_{T, 0}$ is a proper distribution function (df) and $F_{T, 0}((t, \infty) \mid x)=0$ if $H_{1}((t, \infty) \mid x)=0$. If $\widehat{H}_{1}(\cdot \mid x)$ puts a positive mass at the right endpoint of its right-bounded support, then $\widehat{F}_{T, 0}$ is a proper df and $\widehat{F}_{T, 0}((t, \infty) \mid x)=0$ if $\widehat{H}_{1}((t, \infty) \mid x)=0$.

The proof of Lemma 8.1 is provided in the Supplement.

Proof of Proposition 2.1. Equations (2.6) and (2.5) show that $F_{C}(\cdot \mid$ $x)$ is uniquely recovered from $F_{Y, \delta}(\cdot, \cdot \mid x)$. From condition (2.14) and the identity (2.7) it follows that $\beta_{0}$ is identifiable. Finally, the identities (2.11) and (2.12) guarantee that $F_{T, 0}(\cdot \mid x)$ is identifiable.

The next lemma, proved in the Supplement, is used for Theorem 4.1.

Lemma 8.2. Let conditions (4.2), (AC1) and (AC4) hold true. Then,

$$
\begin{gathered}
\sup _{x \in \mathcal{X}} \sup _{t \in(-\infty, \tau(x)]}\left|\widehat{F}_{C}([t, \infty) \mid x)-F_{C}([t, \infty) \mid x)\right|=o_{\mathbb{P}}(1), \\
\sup _{x \in \mathcal{X}} \sup _{t \in(-\infty, \tau(x)]}\left|T_{1}\left(\widehat{H}_{0}, \widehat{H}_{1}\right)(t, x)-T_{1}\left(H_{0}, H_{1}\right)(t, x)\right|=o_{\mathbb{P}}(1),
\end{gathered}
$$

where $T_{1}\left(H_{0}, H_{1}\right)(t, x)=H([t, \infty) \mid x)-\mathbb{P}(T=\infty \mid x) F_{C}([t, \infty) \mid x)$, and $T_{1}\left(\widehat{H}_{0}, \widehat{H}_{1}\right)$ is defined similarly, but with $H_{0}, H_{1}$ replaced by $\widehat{H}_{0}$ and $\widehat{H}_{1}$, respectively. Moreover,

$$
\sup _{x \in \mathcal{X}} \sup _{t \in(-\infty, \tau(x)]}\left|\widehat{F}_{T, 0}([t, \infty) \mid x)-F_{T, 0}([t, \infty) \mid x)\right|=o_{\mathbb{P}}(1) .
$$

Proof of Theorem 4.1. First, we show the consistency of $\widetilde{\beta}$ defined as the maximizer of $\mathcal{L}_{n}(\beta)$, see equation (4.3). Let

$$
\log p(t, \delta, x ; \beta)=\delta \log p_{1}(t, x ; \beta)+(1-\delta) \log p_{0}(t, x ; \beta),
$$

with $p_{1}(t, x ; \beta)=\phi(x, \beta) F_{T, 0}(d t \mid x) F_{C}((t, \infty) \mid x)$ and

$$
p_{0}(t, x ; \beta)=F_{C}(d t \mid x)\left[\phi(x, \beta) F_{T, 0}([t, \infty) \mid x)+1-\phi(x, \beta)\right] .
$$


Following the notation of Gill (1994), here we treat $d t$ not just as the length of a small interval $[t, t+d t)$ but also as the name of the interval itself.

Let us notice that $\log p\left(t, \delta, x ; \beta_{0}\right)=\delta \log H_{1}(d t \mid x)+(1-\delta) \log H_{0}(d t \mid x)$ and the difference $\mathbb{E}[\log p(Y, \delta, X ; \beta)]-\mathbb{E}\left[\log \mathcal{L}_{n}(\beta)\right]$ does not depend on $\beta$. Hence, a minimal condition for guaranteeing the consistency of the likelihood estimation approach is that $\beta_{0}$ is the maximizer of the limit likelihood criterion $\beta \mapsto \mathbb{E}[\log p(Y, \delta, X ; \beta)]$, or equivalently of the $\log$-likelihood ratio $\beta \mapsto \mathbb{E}\left[\log \left\{p(Y, \delta, X ; \beta) / p\left(Y, \delta, X ; \beta_{0}\right)\right\}\right]$. This is proved in the following. By the properties of the likelihood of a Bernoulli random variable given that $Y \in d t$ and $X=x$, for any $\beta \in B$ we have (with the convention $0 / 0=1$ )

$$
\begin{aligned}
& \mathbb{E}\left[\delta \log \frac{\phi(x, \beta) F_{T, 0}(d t \mid x) F_{C}((t, \infty) \mid x)}{H_{1}(d t \mid x)}\right. \\
& \left.+(1-\delta) \log \frac{F_{C}(d t \mid x)\left[\phi(x, \beta) F_{T, 0}([t, \infty) \mid x)+1-\phi(x, \beta)\right]}{H_{0}(d t \mid x)} \mid Y \in d t, X=x\right] \leq 0 .
\end{aligned}
$$

Integrate with respect to $Y$ and $X$ and deduce that $\mathbb{E}[\log p(Y, \delta, X ; \beta)] \leq$ $\mathbb{E}\left[\log p\left(Y, \delta, X ; \beta_{0}\right)\right]$. If $\beta \neq \beta_{0}$ is such that this inequality becomes equality, then necessarily $p_{1}(Y, X ; \beta)=1$ a.s. Then, by the identity $H_{1}(d t \mid x)=$ $F_{C}([t, \infty) \mid x) F_{T}(d t \mid x)$ and condition (2.14), necessarily $\beta=\beta_{0}$. Assumptions (AC3) and (AC4) and condition (2.14) guarantee that $\beta_{0}$ is a well-separated maximum of the continuous map $\beta \mapsto \mathbb{E}\left[\log \mathcal{L}_{n}(\beta)\right], \beta \in B$.

Next, let us rewrite $\log \mathcal{L}_{n}(\beta)=\sum_{i=1}^{n} q\left(Y_{i}, \delta_{i}, X_{i} ; H_{0}, H_{1}, \beta\right)$, where

$$
\begin{aligned}
q\left(t, d, x ; H_{0}, H_{1}, \beta\right)= & d \log \phi(x, \beta) \\
& +(1-d) \log \left\{\phi(x, \beta) F_{T, 0}([t, \infty) \mid x)+1-\phi(x, \beta)\right\} .
\end{aligned}
$$

With our assumptions, it is easy to check that for any $t \in(-\infty, \tau(x)]$, $d \in\{0,1\}, x \in \mathcal{X}$,

$$
\left|q\left(t, d, x ; H_{0}, H_{1}, \beta\right)-q\left(t, d, x ; H_{0}, H_{1}, \beta^{\prime}\right)\right| \leq C\left\|\beta-\beta^{\prime}\right\|^{a}, \quad \forall \beta, \beta^{\prime} \in B,
$$

with $a>0$ from Assumption (AC3) and some constant $C$ depending only on $c_{1}$ from Assumption (AC3) and the positive values $\inf _{x \in \mathcal{X}} H_{1}(\{\tau(x)\} \mid x)$ and $\inf _{x \in \mathcal{X}} H_{0}((\tau(x), \infty) \mid x)$. It follows that the class of functions $\{(t, d, x) \rightarrow$ $\left.q\left(t, d, x ; H_{0}, H_{1}, \beta\right): \beta \in B\right\}$ is Glivenko-Cantelli. Hence,

$$
\sup _{\beta \in B}\left|n^{-1} \log \mathcal{L}_{n}(\beta)-\mathbb{E}\left[n^{-1} \log \mathcal{L}_{n}(\beta)\right]\right|=o_{\mathbb{P}}(1) .
$$

Finally, Lemma 8.2 guarantees that $\sup _{\beta \in B}\left|\log \widehat{L}_{n}(\beta)-\log \mathcal{L}_{n}(\beta)\right|=o_{\mathbb{P}}(n)$. Gathering facts, by asymptotic results as in Section 5.2 of van der Vaart (1998), we deduce that $\widehat{\beta}-\beta_{0}=o_{\mathbb{P}}(1)$. 
Proof of TheOREM 4.2. We show the asymptotic normality of our estimator by verifying the high-level conditions in Theorem 2 in Chen et al. (2003). First of all, for the consistency we refer to Section 4.1, whereas condition (2.1) in Chen et al. (2003) is satisfied by construction. Next, we compute the matrix $\nabla_{\beta} M\left(\beta_{0}, \eta_{0}\right)$. Note that for each $x \in \mathcal{X}$,

$$
\begin{gathered}
\mathbb{E}(\delta \mid X=x)=\phi\left(x, \beta_{0}\right) \int_{(-\infty, \tau(x)]} F_{C}((t, \infty) \mid x) F_{T, 0}(d t \mid x), \\
\mathbb{E}(1-\delta \mid Y=t, X=x)=1-\phi\left(x, \beta_{0}\right) F_{T, 0}((-\infty, t] \mid x) \text { and } \\
\left.\int_{(-\infty, \tau(x)]} F_{C}((t, \infty) \mid x) F_{T, 0}(d t \mid x)=\int_{(-\infty, \tau(x)]} F_{T, 0}(-\infty, t] \mid x\right) F_{C}(d t \mid x) .
\end{gathered}
$$

Hence, we have

$$
\mathbb{E}\left[\frac{\delta}{\phi\left(X, \beta_{0}\right)}-\frac{(1-\delta) T\left(\eta_{0}\right)(Y, X)}{1-\phi\left(X, \beta_{0}\right) T\left(\eta_{0}\right)(Y, X)} \mid X\right]=0 \quad \text { almost surely }
$$

It is now easy to see that $\nabla_{\beta} M\left(\beta_{0}, \eta_{0}\right)$ is given by (4.7). Hence, the matrix is negative definite, and thus condition (2.2) in Chen et al. (2003) is satisfied, as long as the matrix $-\mathbb{E}\left\{\nabla_{\beta} \phi\left(X, \beta_{0}\right) \nabla_{\beta} \phi\left(X, \beta_{0}\right)^{\top}\right\}$ has this property, which is the case thanks assumption (AN3). Thus, we do not need more assumptions than the ones usually considered in with observed binary outcomes.

Next, for condition (2.3), note that using the equation (4.5), we have

$$
\begin{aligned}
& \quad \nabla_{\eta} M\left(\beta, \eta_{0}\right)\left[\eta-\eta_{0}\right] \\
& =-\lim _{\tau \rightarrow 0} \frac{1}{\tau} \mathbb{E}\left[\frac{1-\delta}{\left\{1-\phi(X, \beta) T\left(\eta_{0}\right)(Y, X)\right\}\left\{1-\phi(X, \beta) T\left(\eta_{0}+\tau\left(\eta-\eta_{0}\right)\right)(Y, X)\right\}}\right. \\
& \left.\quad \times\left\{T\left(\eta_{0}+\tau\left(\eta-\eta_{0}\right)\right)(Y, X)-T\left(\eta_{0}\right)(Y, X)\right\} \nabla_{\beta} \phi(X, \beta)\right] .
\end{aligned}
$$

By equation (4.6) and the formulae in section (2.1), we can also write

$$
T\left(\eta_{0}\right)(t, x)=1-\prod_{-\infty<s \leq t}\left\{1-\frac{\eta_{02}(d s, x)}{T_{1}\left(\eta_{0}\right)(s, x)}\right\} .
$$

with $T_{1}(\eta)(t, x)=\eta_{1}(t, x)+\eta_{2}(t, x)-T_{2}(\eta)(x) T_{3}(\eta)(t, x)$,

$$
T_{2}(\eta)(x)=\prod_{t \in \mathbb{R}}\left\{1-\frac{\eta_{2}(d t, x)}{\eta_{1}(t, x)+\eta_{2}(t, x)}\right\},
$$




$$
T_{3}(\eta)(t, x)=\prod_{-\infty<s \leq t}\left\{1-\frac{\eta_{1}(d s, x)}{\eta_{1}(s, x)+\eta_{2}(s, x)}\right\} .
$$

Using Duhamel's formula (see Gill and Johansen 1990) and the dominated convergence theorem, we have

$$
\begin{gathered}
\nabla_{\eta} M\left(\beta, \eta_{0}\right)\left[\eta-\eta_{0}\right]=-\mathbb{E}\left[\frac{(1-\delta) \nabla_{\eta} T\left(\eta_{0}\right)\left[\eta-\eta_{0}\right](Y, X)}{\left\{1-\phi(X, \beta) T\left(\eta_{0}\right)(Y, X)\right\}^{2}} \nabla_{\beta} \phi(X, \beta)\right] \\
=\mathbb{E}\left[\int_{(-\infty, Y)} \frac{1-T\left(\eta_{0}\right)(Y, X)}{T_{1}\left(\eta_{0}\right)(u, X)-\eta_{02}(\{u\}, X)}\right. \\
\times\left\{\left(\eta_{2}-\eta_{02}\right)(d u, X)-\frac{\nabla_{\eta} T_{1}\left(\eta_{0}\right)\left[\eta-\eta_{0}\right](u, X)}{T_{1}\left(\eta_{0}\right)(u, X)} \eta_{02}(d u, X)\right\} \\
\left.\times \frac{1-\delta}{\left\{1-\phi(X, \beta) T\left(\eta_{0}\right)(Y, X)\right\}^{2}} \nabla_{\beta} \phi(X, \beta)\right],
\end{gathered}
$$

where

$$
\begin{aligned}
& \nabla_{\eta} T_{1}\left(\eta_{0}\right)\left[\eta-\eta_{0}\right](y, x)=\left(\eta_{1}-\eta_{01}\right)(y, x)+\left(\eta_{2}-\eta_{02}\right)(y, x) \\
- & \nabla_{\eta} T_{2}\left(\eta_{0}\right)\left[\eta-\eta_{0}\right](x) T_{3}\left(\eta_{0}\right)(y, x)-T_{2}\left(\eta_{0}\right)(x) \nabla_{\eta} T_{3}\left(\eta_{0}\right)\left[\eta-\eta_{0}\right](y, x),
\end{aligned}
$$

and the expressions of $\nabla_{\eta} T_{2}\left(\eta_{0}\right)\left[\eta-\eta_{0}\right](x)$ and $\nabla_{\eta} T_{3}\left(\eta_{0}\right)\left[\eta-\eta_{0}\right](y, x)$ are provided in the Supplementary Material.

Note that the map $y \mapsto T_{1}\left(\eta_{0}\right)(y, x)$ is decreasing on $(-\infty, \tau(x)]$. Moreover, by condition $(4.2), \inf _{x \in \mathcal{X}} T_{1}\left(\eta_{0}\right)(\tau(x), x)>0$. Finally, let us note that by construction for any $y \in(-\infty, \tau(x)), H(\{y\} \mid x)=H_{0}(\{y\} \mid x)+H_{1}(\{y\} \mid$ $x) \geq \mathbb{P}(T=\infty \mid x) F_{C}(\{y\} \mid x)+H_{1}(\{y\} \mid x)$, and thus

$$
\begin{aligned}
H([y, \infty) \mid x)-\mathbb{P}(T= & \infty \mid x) F_{C}([y, \infty) \mid x)-H_{1}(\{y\} \mid x) \\
& \geq H((y, \infty) \mid x)-\mathbb{P}(T=\infty \mid x) F_{C}((y, \infty) \mid x) .
\end{aligned}
$$

Then, $\inf _{x \in \mathcal{X}} \inf _{y \in(-\infty, \tau(x))}\left[T_{1}\left(\eta_{0}\right)(y, x)-H_{1}(\{y\} \mid x)\right]>0$. Hence, all denominators in $\nabla_{\eta} M\left(\beta, \eta_{0}\right)\left[\eta-\eta_{0}\right]$ are bounded away from zero. By tedious but rather elementary Taylor expansions, it now follows that $\nabla_{\eta} M\left(\beta, \eta_{0}\right)[\eta-$ $\eta_{0}$ ] satisfies the second property in condition (2.3) of Theorem 2 in Chen et al. (2003). Similarly, by decomposing $T_{j}(\eta)-T_{j}\left(\eta_{0}\right)-\nabla_{\eta} T_{j}\left(\eta_{0}\right)\left[\eta-\eta_{0}\right]$ $(j=1,2,3)$ using Taylor-type arguments (in $\eta)$, the first property in condition (2.3) is easily seen to hold true.

Next, conditions (2.4) and (2.6) of Theorem 2 in Chen et al. (2003) are satisfied thanks to Assumption (AN4) and because it follows from the above calculations of $\nabla_{\eta} T_{j}\left(\eta_{0}\right)\left[\eta-\eta_{0}\right](j=1,2,3)$ that 
$(8.3) \nabla_{\eta} M\left(\beta_{0}, \eta_{0}\right)\left[\eta-\eta_{0}\right]=\sum_{k=0}^{1} \mathbb{E}\left[\psi_{1 k}(Y, X) \int_{u<Y} \psi_{2 k}(u, X) d\left(\left(\eta_{k}-\eta_{0 k}\right)(u, X)\right)\right]$
$+\sum_{k, \ell \in\{0,1\}} \mathbb{E}\left[\psi_{3 k}(Y, X) \int_{u<Y} \psi_{4 k}(u, X) \psi_{5 k}\left(\left(\eta_{k}-\eta_{0 k}\right)(u, X)\right) d H_{\ell}(u \mid X)\right]$

for certain measurable functions $\psi_{j k}(j=1, \ldots, 5 ; k=0,1)$.

It remains to verify condition (2.5) of Theorem 2 in Chen et al.. Note that

$\left|m\left(t, \delta, x ; \beta_{2}, \eta_{2}\right)-m\left(t, \delta, x ; \beta_{1}, \eta_{1}\right)\right| \leq C_{1}(t, \delta, x)\left\|\beta_{2}-\beta_{1}\right\|+C_{2}(t, \delta, x)\left\|\eta_{2}-\eta_{1}\right\|_{\mathcal{H}}$

for some $C_{j}$ satisfying $\mathbb{E}\left[C_{j}^{2}(Y, \delta, X)\right]<\infty(j=1,2)$, and hence (2.5) follows from our assumption (AN5) and Theorem 3 in Chen et al. (2003).

Acknowledgements. V. Patilea acknowledges support from the research program New Challenges for New Data of Fondation du Risque and LCL. I. Van Keilegom acknowledges support from the European Research Council (2016-2021, Horizon 2020/ERC grant agreement No. 694409).

\section{SUPPLEMENT}

The supplement is organized as follows. Appendix A contains the proof of the i.i.d. representation (4.9), Appendix B shows the calculation of the efficient score function, Appendix $\mathrm{C}$ contains details on the maximization procedure used in the simulations, Appendix D collects additional simulation results, Appendix E shows the analysis of an additional dataset, and Appendix F collects proofs of technical lemmas and equations.

\section{REFERENCES}

[1] Akritas, M.G. \& Van Keilegom, I. (2001). Nonparametric estimation of the residual distribution. Scand. J. Statist. 28, 549-568.

[2] Amico, M., Legrand, C. \& Van Keilegom, I. (2018). The single-index/Cox mixture cure model Biometrics (to appear).

[3] Amico, M. \& Van Keilegom, I. (2018). Cure models in survival analysis. Ann. Rev. Statist. Applic., 5, 311-342.

[4] BoAg, J.W. (1949). Maximum likelihood estimates of the proportion of patients cured by cancer therapy. J. Roy. Statist. Soc. - Series B 11, 15-53.

[5] Chen, X., Linton, O. \& Van Keilegom, I. (2003). Estimation of semiparametric models when the criterion function is not smooth. Econometrica 71, 1591-1608.

[6] FAng, H.B., Li, G. \& Sun, J. (2005). Maximum likelihood estimation in a semiparametric logistic/proportional-hazards mixture model. Scand. J. Statist. 32, 59-75.

[7] Farewell, V.T. (1982). The use of mixture models for the analysis of survival data with long-term survivors. Biometrics 38, 1041-1046.

[8] GILL, R.D. (1994). Lectures on survival analysis. Lectures on probability theory: Ecole d'été de probabilités de Saint-Flour XXII. Lecture notes in mathematics 1581. Springer. 
[9] Gill, R.D. \& Johansen, S. (1990). A survey of product-integration with a view toward application in survival analysis. Ann. Statist. 18, 1501-1555.

[10] Kuk, A.Y.C. \& Chen, C.-H. (1992). A mixture model combining logistic regression with proportional hazards regression. Biometrika 79, 531-541.

[11] Li, Q., Lin, J. \& RACine, J.S. (2013). Optimal bandwidth selection for nonparametric conditional distribution and quantile functions. J. Buss. Econ. Statist. 31, 57-65.

[12] López-Cheda, A., Cao, R., Jácome M.A. \& Van Keilegom, I. (2017). Nonparametric incidence estimation and bootstrap bandwidth selection in mixture cure models. Comput. Statist. Data Anal. 105, 144-165.

[13] Lopez, O. (2011). Nonparametric estimation of the multivariate distribution function in a censored regression model with applications. Commun. Stat. - Theory Meth. 40, 2639-2660.

[14] LU, W. (2008). Maximum likelihood estimation in the proportional hazards cure model. Ann. Inst. Stat. Math. 60, 545-574.

[15] MeEker, W.Q. (1987). Limited failure population life tests: Application to integrated circuit reliability. Technometrics 29, 51-65.

[16] Oтhus, M., Li, Y. \& Tiwari, R.C. (2009). A class of semiparametric mixture cure survival models with dependent censoring. J. Amer. Statist. Assoc. 104, 1241-1250.

[17] Peng, Y. \& Taylor, J.M.G. (2014). Cure models. In: Klein, J., van Houwelingen, H., Ibrahim, J. G., and Scheike, T. H., eds, Handbook of Survival Analysis, Handbooks of Modern Statistical Methods series, ch 6, p 113-134. Chapman \& Hall, Boca Raton, FL, USA.

[18] Schmidt, P. \& Witte, A.D. (1989). Predicting criminal recidivism using split population survival time models. J. Econometrics 40, 141-159.

[19] Stone, C.J. (1977). Consistent Nonparametric Regression. Ann. Statist. 5, 595-620.

[20] Sy, J.P. \& TAYLOR, J.M.G. (2000). Estimation in a Cox proportional hazards cure model. Biometrics 56, 227-236.

[21] TAYLOR, J.M.G. (1995). Semi-parametric estimation in failure time mixture models. Biometrics 51, 899-907.

[22] VAN Der VaArT, A.D. (1998). Asymptotic Statistics. Cambridge University Press.

[23] Wang, Y., Klijn, J.G.M., Sieuwerts, A.M., Look, M.P., Yang, F., Talantov, D., Timmermans, M., Meijet-van Gelder, M.E.M., Yu, J., Jatkoe, T., Berns, E.M.J.J., Atkins, D. \& Foekens, J.A. (2005). Gene-expression profiles to predict distant metastasis of lymph-node-negative primary breast cancer. The Lancet $\mathbf{3 6 5}$, $671-679$.

[24] Xu, J. \& Peng, Y. (2014). Nonparametric cure rate estimation with covariates. Canad. J. Statist. 42, 1-17.

CREST (UMR 9194), ENSAI

CAMPus de Ker-LanN

35172 BRUZ, FRANCE

E-MAIL: patilea@ensai.fr
ORSTAT, KU LEUVEN

NAAMSESTRAAT 69

3000 Leuven, BELGIUM

E-MAIL: ingrid.vankeilegom@kuleuven.be 